\title{
Hypoxia Enhanced Glioblastoma Resistance to Erastin-Induced Ferroptosis by Up-Regulating GPX4 via PI3K/AKT/HIF-1a Axis
}

\section{Shicheng Sun}

Shandong Provincial Hospital

Changfa Guo

Shandong Provincial Hospital

Bo Pang

Shandong University Qilu Hospital

Taihong Gao

Shandong Provincial Hospital

Xiangsheng Su

Shandong Provincial Hospital

Dong He

Shandong Provincial Hospital

Rui Zhang ( $\nabla$ rui.zhang@sdu.edu.cn )

Shandong Provincial Hospital https://orcid.org/0000-0003-2368-599X

Qi Pang

Shandong Provincial Hospital

\section{Research Article}

Keywords: GPX4, HIF-1a, Hypoxia, ferroptosis, Glioblastoma

Posted Date: December 8th, 2021

DOI: https://doi.org/10.21203/rs.3.rs-1122453/v1

License: (1) (1) This work is licensed under a Creative Commons Attribution 4.0 International License.

Read Full License 


\section{Abstract \\ Background}

Glioblastoma is the deadliest type of primary brain tumor with a high rate of recurrence and treatment resistance. Hypoxia contributed much to radiotherapy resistance and chemoresistance of cancer. Ferroptosis is a nonapoptotic, oxidative cell death and identified as a potential anticancer mechanism in recent years. Erastin acts as a ferroptosis activator and shows a potential role in tumor treatment but the relationship between hypoxia and erastin resistance in glioblastoma has not been explained. This study aimed to investigate the role and underlying mechanism of hypoxia in erastin-induced ferroptosis in glioblastoma.

\section{Methods}

Cell proliferation and viability were determined by Cell Counting Kit-8 (CCK-8) assay, flow cytometry, TUNEL assays, and clone formation assay. Lipid peroxides level was analyzed by Malondialdehyde (MDA) assay and flow cytometry using C11-BODIPY dye. The correlation between HIF-1a and GPX4 expression was detected in data from the TCGA database and was determined by ChIP-qPCR assay and luciferase reporter assay. Subcutaneous xenograft and orthotopic xenograft models were established to test our findings in vivo.

\section{Results}

Hypoxia for at least 16 hours significantly suppressed erastin-induced ferroptosis by up-regulating glutathione peroxidase 4 (GPX4) expression in U87 and U251 cells. Hypoxia promotes GPX4 expression via enhancing the PI3K/AKT/HIF-1a pathway. Mechanistically, HIF-1a directly bound to the GPX4 gene promoter region and promoted GPX4 transcription. AKT inhibitor MK-2206 and HIF-1a inhibitor PX-478 could significantly reverse the effect. Besides, under normoxia, PX-478 could induce a higher lipid peroxidation level by decreasing GPX4 expression in U87 and U251 cells but cannot induced cell death directly, and it could significantly enhance the tumor cell killing effect of erastin. In vivo, combination of PX-478 and erastin had a coordinated intensification effect on anticancer activity uncovered by subcutaneous xenograft and orthotopic xenograft mouse models.

\section{Conclusions}

Hypoxia enhanced glioblastoma resistance to erastin-induced ferroptosis by activating PI3K/AKT/HIF-1a pathway and promoting GPX4 expression in a transcriptional regulation way. Combination therapy of PX478 and erastin may be a potential strategy against glioblastoma. 


\section{Background}

Glioma is most common primary malignant brain tumor in adults, in which glioblastoma is the most malignant and aggressive form [1]. Glioblastoma is an anaplastic, poorly differentiated malignant tumor with a peak of incidence between 45 and 70 years [2]. Despite recent advances in diverse therapy for glioblastoma incorporating surgery, radiotherapy, chemotherapy, the median survival is only about 15 months [3-4]. Thus, more efficient therapeutic strategies should be developed.

The tumor microenvironment (TME) refers to the local biological environment in which solid tumors are located [5]. The TME often displays at least some degree of hypoxia [6]. Coincidentally, glioblastoma undergoes malignant progression under hypoxia condition [7]. Tumor hypoxia is generated by irregular and tortuous vasculature formed within solid tumors, hence causing a poor delivery of oxygen to cells. Hypoxia is associated with malignancy and tumor aggressiveness, by increasing tumor cell proliferation, metastasis [8]. Besides, hypoxia has been associated with resistance to radiotherapy and several chemotherapies. For one thing, hypoxia contributes to radioresistance by controlling several cellular processes including cell cycle [9], apoptosis and senescence [10], creation of reactive oxygen species [11], invasion and cancer cell stemness [12]. For another, hypoxia enhanced the resistance to temozolomide by different mechanisms including inducing ferritin light chain [13], regulating glioma stem cells properties [14], mediating ATP-binding cassette proteins [15]. Hypoxia-inducible factor (HIF) is upregulated in the tumor hypoxia microenvironment, in which HIF-1 $a$ and HIF-2 $a$ are considered the main regulators of the hypoxia response [16]. Under hypoxia, HIF-1a is stabilized and translocated into the nucleus to play roles in transcription [17]. As a selective HIF-1a inhibitor, PX-478 interferes with HIF-1a and induces cell cycle arrest in cancer cells [18-20].

Ferroptosis is a form of nonapoptotic, oxidative cell death that plays an essential role in various diseases, including cranial trauma, neuronal diseases, and brain tumors [21]. Lipid peroxidation underlies the mechanism of ferroptosis [22]. GPX4 and ferroptosis suppressor protein 1 constitute two major ferroptosis defense systems through the inhibition of lipid peroxidation [23]. Erastin as the ferroptosis activator can not only cause tumor cells death, but also increase the sensitivity of tumor cells to chemotherapeutic drugs by coordinating with chemotherapy drugs [24]. For example, erastin has been proved to enhance the sensitivity of glioma cells to temozolomide, which showed the potential role of erastin in tumor treatment [25].

To date, the relationship between hypoxia and erastin-induced ferroptosis has not been reported in glioblastoma. In the present study, we investigated the role of hypoxia in erastin-induced ferroptosis in glioblastoma cell lines and determined the involved molecular mechanisms. Our study not only identified a novel mechanism of erastin resistance but also suggested a novel treatment thought by combination of ferroptosis activator and HIF-1a inhibitor.

\section{Material And Methods}




\section{Cell lines}

Human glioblastoma cell lines U87 and U251 were directly purchased from Cell Bank of Type Culture Collection of Chinese Academy of Sciences (Shanghai, China). These cells were cultured in high glucose Dulbecco's modified Eagle's medium (DMEM, Gibco, USA) with 10\% fetal bovine serum (FBS, Biological, Industries) and $1 \%$ penicillin-streptomycin (10378016, Invitrogen, USA) at $37^{\circ} \mathrm{C}$ with $5 \% \mathrm{CO} 2$.

\section{Cell growth and cell viability assay}

Cell growth was determined using a Cell Counting Kit-8 (CCK-8) assay kit (HY-K0301, MCE, Shanghai, China). U87 and U251 cells were seeded into 96 -well plates for $0,24,48$, and $72 \mathrm{~h}$ at a density of 3000 cells per well. Then, $10 \mu \mathrm{l}$ CCK-8 solution was added to each well and incubated with the cells for $2 \mathrm{~h}$. Absorbance (Abs) was detected at $450 \mathrm{~nm}$ using a microplate reader (Bio-Rad, Hercules, CA, USA).

For cell viability assay, cells were seeded at 1000 cells per well in 96-well plates with fresh medium and analyzed by using the CCK-8 assay kit according to the above instructions. The experimental group contained erastin (HY-15763, MCE, Shanghai, China), and the cells treated with DMSO were used as the control group. Herein, cell viability = (Abs of the experimental group-Abs of the blank group)/ (Abs of the control group-Abs of the blank group) $\times 100 \%$.

\section{Vector construction and transduction}

Stable overexpression of GPX4 and the control genes in cells were achieved by transfection with lentivirus synthesized by Genomeditech (Shanghai, China). Cells were infected with Lv-GPX4 or negative control lentivirus vectors. GPX4 siRNA was used to silence GPX4 gene expression. The target sequence of GPX4 was 5'-CCGATACGCTGAGTGGGT-3'. A scrambled siRNA targeting 5'TTCTCCGAACGTGTCACGT-3' was used as a negative control.

\section{Half-maximal inhibitory concentration assay (IC50)}

The cells were planted in 96 -well plates at $1.0 \times 104$ cells per well. The corresponding concentrations of erastin were given to cells for $24 \mathrm{~h}$. After $24 \mathrm{~h}$, CCK-8 was used to measure erastin sensitivity at $450 \mathrm{~nm}$ after incubating for $2 \mathrm{~h}$ at $37^{\circ} \mathrm{C}$.

\section{RNA extraction and quantitative real-time PCR array}

Total RNA was extracted from cultured cells using a Total RNA extraction kit (RC112-01, Vazyme Biotech, Nanjing, China). The concentration and purity of RNA were measured by the absorbance at $260 \mathrm{~nm}$ and the ratio of 260/280nm in NanoDrop ND-1000 (NanoDrop, Wilmington, DE, USA). Total RNA from each sample was reversely transcribed using an all-in-one cDNA synthesis superMix (R333-01, Vazyme Biotech, Nanjing, China). SYBR Green PCR kit (R311-02, Vazyme Biotech, Nanjing, China) was used for real-time PCR. The primers of related genes are listed in Table S1. Quantitative PCR arrays are designed to analyze a panel of ferroptosis-related genes in human glioblastoma cell line U87 and U251 following the instructions of the manufacturer (Wcgene Biotechnology Corporation, China). 


\section{Western blot analysis}

Protein samples from cells were lysed in Cell Lysis Buffer (P0013C, Beyotime, shanghai, China) containing protease/phosphates inhibitors (P1050, Beyotime Biotechnology, Shanghai, China). The concentration of the protein homogenates was determined using the BCA assay Kit (P0012, Beyotime, shanghai, China). Equal volumes of protein samples were separated by SDS-poly acrylamide gel electrophoresis and electro-transferred to PVDF membranes (Millipore, Billerica, MA, USA). After blocked with $5 \%$ non-fat milk dissolved in TBST (10 mM Tris, $150 \mathrm{mM} \mathrm{NaCl}$, and $0.1 \%$ Tween-20; $\mathrm{pH} 7.6)$, for $2 \mathrm{~h}$ at room temperature, the membranes were incubated with the primary antibodies overnight at $4^{\circ} \mathrm{C}$.

Thereafter, membranes were incubated with the secondary antibodies coupled to horseradish peroxidase (HRP) for $1 \mathrm{~h}$ at room temperature. Protein bands were visualized with a super-sensitive electrochemiluminescence (ECL) reagent (WBULS0100, Millipore, USA). The primary antibodies were rabbit polyclonal anti-GPX4 (ab40993, Abcam, USA), rabbit monoclonal anti-STEAP3 (ab151566, Abcam, USA), rabbit monoclonal anti-CA9 (ab108351, Abcam, USA), rabbit polyclonal anti- $\beta$-actin (ab8227, Abcam, USA), rabbit monoclonal anti-HIF-1a (ab179483, Abcam, USA).

\section{Immunofluorescence (IF) and Immunohistochemistry (IHC)}

U87 and U251 cells were grown on cover slides, fixed, blocked with 3\% BSA, and permeabilized with PBS containing $0.1 \% \mathrm{w} / \mathrm{v}$ Triton $\mathrm{X}-100$. The mouse brain of the orthotopic intracranial mouse model was cut in the thickness of $20 \mu \mathrm{m}$ by a frozen section and blocked with $3 \%$ BSA. For protein detection, the primary antibodies used have been already described in reagents and antibodies section. For IF, Secondary antibodies were goat IgG anti-rabbit conjugated to a fluorochrome. Nuclei were stained by 4,6-diamidino2phenylindole (DAPI). Image acquisition was performed on ImageXpress Micro Confocal Devices. IHC staining was performed by using Immunofluorescence Two-Step Test Kit (PV-9000, ZSGB-BIO, Beijing China) according to the manufacturer's instructions. Image acquisition was performed on an OLYMPUS BX63 microscope.

\section{Clone formation assay}

U87 and U251 cells ( $3 \times 102$ cells/plate) were seeded in $35 \mathrm{~mm}$ plates and grew for 2 weeks before being fixed with $4 \%$ paraformaldehyde for $15 \mathrm{~min}$ at room temperature. The cells were washed twice with PBS and stained with Crystal Violet Staining Solution (C0121, Beyotime Biotechnology, Shanghai, China).

\section{TUNEL assay}

For the terminal deoxynucleotidyl transferase (TdT) dUTP nick-end labeling (TUNEL) assays, U87 and U251 cells were seeded into 96-well plates at $1 \times 10^{4}$ cells per well treated with saline, PX-478, erastin or PX-478 combined with erastin for 6 hours. Cells were then stained with the TMR (red) Tunel Cell Apoptosis Detection Kit (G1502, Servicebio, China) according to the manufacturer's protocol. Images were acquired with a ImageXpress Micro Confocal (Molecular Devices, USA) and the percentage of TUNELpositive cells was calculated.

\section{Cell apoptosis analysis by flow cytometry}


Cell apoptosis was measured by the Annexin V-fluorescein isothiocyanate (FITC)/propidium iodide (PI) kit (BD556547, BD Bioscience, USA). Briefly, U87 and U251 cells were seeded into 6-well plates treated with saline, PX-478, erastin or PX-478 combined with erastin for 6 hours, and then the cells were collected and washed twice with cold PBS. Next, the cells were resuspended in $1 \times$ binding buffer at a concentration of $1 \times 10^{6}$ cells per $\mathrm{ml}$ and transferred $100 \mu \mathrm{l}$ of the solution $\left(1 \times 10^{5}\right.$ cells $)$ to a $5 \mathrm{ml}$ culture tube. Incubated with $5 \mu \mathrm{L}$ Annexin V-FITC and $10 \mu \mathrm{L} \mathrm{PI}$ for $15 \mathrm{~min}$ at (RT) $25^{\circ} \mathrm{C}$ in the dark and then add $400 \mu \mathrm{l}$ of $1 \times$ Binding Buffer to each tube. The results were analyzed using a FACS Calibur or an EPICS XL flow cytometer (BD Biosciences).

\section{ChIP-qPCR assay}

To determine whether HIF-1 a can bind to the GPX4 promoter, a ChIP-qPCR assay was used. Briefly, cells were cross-linked using $1 \%$ formaldehyde, followed by scraping into ice-cold PBS with protease inhibitors. Next, the cells were collected and resuspended in lysis buffer $(20 \mathrm{mM} \mathrm{HEPES}, \mathrm{pH}=7.9,420 \mathrm{mM} \mathrm{NaCl}, 0.2$ mM EDTA, $0.5 \%$ NP-40, 25\% glycerol, $1.5 \mathrm{mM} \mathrm{MgCl} 2$ ), followed by several periods of sonication. One-third of the cell extract was put as input sample and two-thirds of the cell extract was used as substrate for immunoprecipitation with anti-HIF-1a antibody. Next, $5 \mathrm{MNaCl}$ was used to reverse the cross-linking after which the eluted DNA was extracted for PCR analysis. The primer sequences of GPX4 promoter were listed in Table S1.

\section{TCGA data analysis}

mRNA expression data from the TCGA database was used to assess correlation between HIF-1a and GPX4 expression. The analyses were performed using the online analysis software named cBioPortal (http://www.cbioportal.org). We used Whole-exome and/or whole-genome sequencing of 257 tumor/normal pairs in glioblastoma (TCGA, Cell 2013) study [26].

\section{Luciferase reporter assay}

To investigate the role of interaction between HIF-1a and GPX4, we constructed GPX4 promoter-driven luciferase reporter plasmids and transfected into U87 and U251 cells. Cells were treated with PX-478 (HY10231, MCE, Shanghai, China), or DMSO and exposed to $1 \% 02$ for $16 \mathrm{~h}$. The ratios of firefly and Renilla luciferase activities were determined $48 \mathrm{~h}$ post-transfection using a Dual-Luciferase Assay kit (E1910, Promega, USA) according to the instructions of the manufacturer.

\section{Malondialdehyde (MDA) assay}

The relative MDA concentration in cell lysate was assessed using an MDA Assay kit (BC0025, Servicebio, China) according to the manufacturer's instructions [27]. Measure the absorbance at $532 \mathrm{~nm}$ using a microplate reader.

\section{Subcutaneous xenograft and orthotopic xenograft mouse model}


All experimental animal procedures were conducted strictly by the Guide for the Care and Use of Laboratory Animals and approved by the Animal Care and Use Committee of the Shandong provincial hospital affiliated to Shandong University. The male BALB/c nude mice were randomized divide into 5 groups in a blinded manner, each group including six 4-weeks-old nude mice. In the subcutaneous xenograft model, $1 \times 10^{6} \mathrm{U} 87$ cells were subcutaneously injected in the right flanks of nude mice. At the second week after injection, control group was intraperitoneally administered DMSO meanwhile oral given saline twice a day; erastin group was intraperitoneally administered erastin $(5 \mathrm{mg} / \mathrm{kg})$ meanwhile oral given saline twice a day; PX-478 group was intraperitoneally administered DMSO meanwhile oral given PX-478 $(100 \mathrm{mg} / \mathrm{kg})$ twice a day; PX478+erastin group was intraperitoneally administered erastin (5 mg/kg) meanwhile oral given PX-478 (100 mg/kg) twice a day; PX-478+Lv-GPX4+erastin group was subcutaneously implanted $1 \times 10^{6}$ Lv-GPX4 transfected U87 cells and was intraperitoneally administered erastin $(5 \mathrm{mg} / \mathrm{kg})$ meanwhile oral given PX-478 $(100 \mathrm{mg} / \mathrm{kg})$ twice a day. At day 23 after implantation, subcutaneous tumors were collected, fixed with $4 \%$ paraformaldehyde, and sectioned for measurement.

In the orthotopic xenograft mouse model, each mouse was intracranially injected with $1 \times 10^{5}$ luciferase

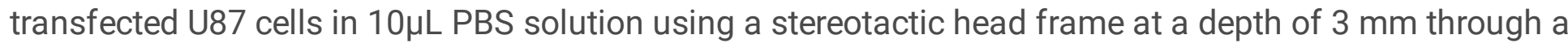
bur hole placed $2 \mathrm{~mm}$ lateral and $2 \mathrm{~mm}$ Posterior to the bregma. At the second week after injection, mice received erastin $(5 \mathrm{mg} / \mathrm{kg})$ and PX-478 $(100 \mathrm{mg} / \mathrm{kg})$ treatment the same as subcutaneous xenograft model for 2 weeks. In vivo tumor growth was monitored with a Xenogen IVIS Spectrum system (PerkinElmer) every week after implantation.

\section{Lipid peroxides assay}

Lipid peroxides level was analyzed by flow cytometry using C11-BODIPY dye. U87 and U251 cells were seeded into 6-well plates at $3 \times 10^{5}$ cells per well and treated with ferroptosis inducer erastin as well as ferroptosis inhibitor ferrostatin-1 (HY-100579, MCE, Shanghai, China). The culture medium was replaced with $1 \mathrm{ml}$ medium containing $10 \mu \mathrm{M}$ of C11-BODIPY (D3861, Thermo Fisher Scientific, USA), and then cells were incubated for $30 \mathrm{~min}$ in a humidified incubator (at $37^{\circ} \mathrm{C}, 5 \% \mathrm{CO}$ ). Media then were removed and washed cells with PBS three times. For measurement by using fluorescence microscope, Images were acquired with a ImageXpress Micro Confocal (Molecular Devices, USA). For measurement by using flow cytometry, Cells were harvested in $15 \mathrm{ml}$ tubes and resuspended in $500 \mu \mathrm{l}$ of PBS. The cell suspension was filtered through cell strainer ( $0.4 \mu \mathrm{m}$ nylon mesh). The results were analyzed using a FACS Calibur or an EPICS XL flow cytometer (BD Biosciences).

\section{Transmission electron microscopy assay}

Transmission electron microscopy analyses were conducted [28]. Briefly, U87 and U251 cells were seeded into 6-well plates at $3 \times 10^{5}$ cells per well and exposed to saline, PX-478 or Iv-GPX4 combined with PX478 for $6 \mathrm{~h}$. After that, cells were collected, washed three times with PBS, and fixed with $2.5 \%$ glutaraldehyde. Samples were then pretreated according to standard procedures including staining, dehydration, embedding, and slicing to obtain ultra-thin sections. During the analysis, images were acquired using a HITACHIH-7000 transmission electron microscope (TEM; Hitachi, Tokyo, Japan). 


\section{Statistical analysis}

Data were presented as mean values \pm standard deviation (SD) from at least three experiments. Student's t-test used to analyze the differences between two groups and One-way analysis of variance (ANOVA) used for the comparison among three or more groups were conducted using GraphPad Prism 8 (GraphPad Software, CA, USA), SPSS (IBM, NY, USA). p-values less than 0.05 were considered statistically significant $\left({ }^{\star} p<0.05,{ }^{\star \star} p<0.01\right)$.

\section{Results}

\section{Hypoxia protected glioblastoma cells against erastin- induced ferroptosis}

Hypoxia condition $\left(1 \% \mathrm{O}_{2}, 5 \% \mathrm{CO}_{2}, 94 \% \mathrm{~N}_{2}\right)$ was a crucial factor that contributed to the aggressive behavior of glioblastoma [29]. We first evaluated whether hypoxia could affect the viability of U87 and U251 cells by CCK-8 cell viability assay. As depicted in Fig. 1A, Hypoxia condition promoted U87 and U251 cell proliferation significantly. Then we explored the relationship between hypoxia and erastin resistance over a series of time points in U87 and U251 cells. Cell resistance was measured by the ratio (cells exposed to erastin (10 $\mu \mathrm{M}, 6$ hours) / cells exposed to DMSO). We found hypoxia for at least 16 hours could induce a stronger resistance to erastin (Fig. 1B). Then we explored the cell viability at different concentrations of erastin for $6 \mathrm{~h}$. We found glioblastoma cells under hypoxic environment became less sensitive to erastin (Fig. 1C). The half-maximal inhibitory concentrations (IC50) of hypoxic U87 and U251 cells to erastin were obviously high than that of normoxic cells (Fig. 1D). Furthermore, the lipid peroxidation level was measured by flow immunolabeling using C11-BODIPY probe. Results showed that high lipid peroxidation level induced by erastin ( $10 \mu \mathrm{M}, 6$ hours) was decreased significantly by hypoxic incubation ( $1 \% \mathrm{O}_{2}, 16$ hours) and ferroptosis inhibitor ferrostatin-1 (fer- $1,0.5 \mu \mathrm{M}, 6$ hours) (Fig. 1E). Moreover, we explored the effect of hypoxia and erastin on cell colony formation ability. We found hypoxia condition and Fer-1 apparently enhanced the cell colony formation ability when treating cells with $10 \mu \mathrm{M}$ erastin for 2 weeks (Fig. 1F). Together, these results suggested that hypoxic treatment for at least 16 hours decreased the sensitivity of glioblastoma cells to erastin.

\section{GPX4 acted as a potential effector molecular of hypoxia}

To define potential genes that played vital roles in erastin-resistance under hypoxic condition, an ferroptosis profiling qPCR array was employed by comparing cells under hypoxic condition for 16 hours and normoxic condition. Each group was repeated four times (Fig. 2A). Heterogeneity of tumor cells resulted in diverse qPCR array results between U87 and U251 cells. Considering that both cell lines showed similar decreased sensitivity to erastin, we identified genes that changed significantly in both cell lines as our potential targets. Three genes were picked out, namely GPX4, STEAP3 metalloreductase (STEAP3), carbonic anhydrase 9 (CA9) (Fig. 2B). Next, we detected the protein expression level of the three genes over a series of time points by western blot. We found the time course of GPX4 protein 
expression confirmed the trends of enhanced erastin-resistance, but not STEAP3 and CA9. At the 16th hour under hypoxia, GPX4 protein level increased significantly with the enhancement of resistance to erastin (Fig. 2C). To further verify this observation, we assessed GPX4 protein location and expression level via cellular immunofluorescence. The results showed higher GPX4 protein level after at least 16 hours hypoxic incubation and no apparent location changes at several indicated time points (Fig. 2D). Taken all together, GPX4 acted as a potential effector molecular of hypoxia and we identified GPX4 as a target for further investigation.

\section{Hypoxia enhanced the resistance to erastin via increasing GPX4 expression}

To investigate the role of GPX4 in hypoxia-induced resistance to erastin, we used GPX4 siRNA to inhibit GPX4 gene expression in U87 and U251 cells. Firstly, we analyzed the efficacy of GPX4 knockout (GPX4 $\mathrm{KO}$ ). Western blot showed siRNA can significantly inhibit the protein expression level of GPX4 (Fig. 3A). Quantitative analysis results were showed in figure S1. CCK-8 cell viability assay showed GPX4 knockout did not influence the cell proliferation rate of glioblastoma cells (Fig. 3B). Cytotoxicity experiments showed GPX4 knockout could reverse the enhanced erastin-resistance induced by hypoxia, meanwhile, GPX4 knockout under normoxia could cause cells to be more sensitive to erastin (Fig. 3C).

The IC50 of GPX4 knockout cells was obviously lower than that of scramble cells under hypoxic condition (Fig. 3D). Next, we explored whether GPX4 knockout could influence the cell colony formation ability. We found GPX4 knockout could reverse the enhanced cell colony formation ability induced by hypoxia when treating with erastin and GPX4 knockout under normoxia led to less colony formation when treating with erastin (Fig. 3E). In short, we identified GPX4 as one of the most vital gene which regulated the ferroptosis defense ability of glioblastoma cells under hypoxic environment.

\section{PI3K/AKT/HIF-1a pathway involved in the up-regulation of GPX4 induced by hypoxia}

$\mathrm{PI}$ 3K/AKT pathway had been reported to participate in malignant progression of glioblastoma and considered as an important regulator of ferroptosis [30-31]. HIF-1 a was a well-known transcription factors that play an important role in the early stage of hypoxia [16]. Thereby, we hypothesized that the increased GPX4 expression might owing to the activation of the PI3K/AKT/HIF-1a pathway. To prove our hypothesis, we explored the protein changes in the PI3K/AKT/HIF-1a pathway by western blot. Results indicated that hypoxia incubation for 16 hours obviously activated PI3K, phosphorylated AKT, HIF-1a and GPX4. Treatment with the AKT inhibitor MK-2206 along with hypoxia incubation significantly attenuated the expression level of p- AKT, HIF-1a and GPX4 (Fig. 4A). Quantitative analysis results were showed in figure S2. Then we employed HIF-1a inhibitor PX-478 to further test the function of HIF-1a. Results showed PX-478 can markedly decreased HIF-1a and GPX4 protein levels (Fig. 4B). Then we analyzed public data [26] and found the expression of GPX4 was positively correlated with HIF-1a in 257 glioblastoma tumor samples (Fig. 4C). To understand the molecular mechanism underlying the regulation of GPX4 by HIF-1a, we performed chromatin immunoprecipitation (ChIP) with an anti-HIF-1a 
antibody to test whether HIF-1a can bind to the GPX4 promoter region. We designed three pairs of primer to detect different regions of GPX4 promoter respectively and uncovered that HIF- $1 \mathrm{a}$ can indeed bind to the GPX4 promoter region -131 bp to $1 \mathrm{bp}$ (Fig. 4D). Therefore, we transfected GPX4 promoter-driven luciferase reporter plasmid into U87 and U251 cells and tested relative luciferase activity under normoxia condition, hypoxia condition, and hypoxia with PX-478 condition. Hypoxia group exhibited increased luciferase activity and hypoxia with PX-478 group resulted in the decrease of luciferase activity (Fig. 4E). Finally, we explored whether the erastin-resistance ability induced by hypoxia was related to HIF-1a. We found inhibition of HIF-1a by PX-478 can reverse the enhanced erastin-resistance (Fig. 4F) and elevated IC50 (Fig. 4G) induced by hypoxia in both cell lines.

\section{HIF-1a inhibitor PX-478 induced lipid peroxidation in glioblastoma cells}

Given that ferroptosis resistance can be induced by hypoxia mentioned above and lipid peroxidation underlay the mechanism of ferroptosis [22]. we determined whether suppression of HIF-1a induced lipid peroxidation in glioblastoma cells. Indeed, we utilized C11-BODIPY probe and observed that HIF-1a inhibitor PX-478 $(20 \mu \mathrm{M})$ significantly increased lipid peroxidation level in the glioblastoma cells. To determine whether GPX4, as a downstream of HIF-1a, could reverse lipid peroxidation level induced by PX-478, the glioblastoma cells were transfected with lentivirus which overexpressed human GPX4 (PX478+ Lv-GPX4 group). As expected, the lipid peroxidation level was decreased in the PX-478+ Iv-GPX4 group (Fig. 5A). As depicted in Figure. 5B, the ratios of C11-BODIPY+ cells in PX-478 treatment group were $12.7 \%$ and $21.4 \%$ in U87 and U251 cell lines respectively, and those in control group were $3.55 \%$ and $4.43 \%$, indicating the lipid peroxidation level after treating with PX-478 was significantly increased. Importantly, combination of PX-478 and Iv-GPX4 resulted in a low lipid peroxidation level, the ratios of C11-BODIPY+ cells were $4.62 \%$ and $5.15 \%$ in U87 and U251 cells respectively. Furthermore, we confirmed it by measuring malondialdehyde (MDA), a product of lipid oxidized damage. Results showed that the lipid oxidized damage level was increased after treating with PX-478 and reversed in the presence of LVGPX4 (Fig. 5C). Transmission electron microscopy revealed that PX-478 treated cancer cells exhibited shrunken mitochondria with enhanced membrane density, which were morphologic features of ferroptosis. Black arrays represented normal mitochondrial. White arrays represented shrunken and high membrane density mitochondrial. GPX4 overexpression could reverse it (Fig. 5D). Then, we studied the potential role of PX-478 in erastin-induced ferroptosis in normoxic condition. We found that treatment with PX-478 alone could not cause cell death, which suggested that a modest degree of lipid peroxidation induced by PX-478 will not result in cell death directly. We examined the combined effect of erastin and PX-478. Results indicated that the combined medication had a significant higher cell death rate than erastin alone uncovered by flow cytometry analysis and TUNEL staining, meanwhile, GPX4 overexpression blocked the cytotoxic effect (Fig. 5E, F).

\section{PX-478 enhanced the anticancer activity of erastin in vivo}

To further investigate the role of PX-478 in promoting erastin sensitivity in vivo, we established subcutaneous xenograft and orthotopic xenograft mouse model of glioblastoma as described in 
methods. In both subcutaneous xenograft and orthotopic xenograft model, erastin $(5 \mathrm{mg} / \mathrm{kg}$ ) was intraperitoneal injected twice a day and PX-478 $(100 \mathrm{mg} / \mathrm{kg})$ was oral given twice a day for 2 weeks. Detailed experimental process was put in Materials and Methods. Compared with control group (DMSO intraperitoneal injection and saline oral given), erastin group (erastin intraperitoneal injection and saline oral given) exhibited a certain degree of anticancer effect but not PX-478 group (DMSO intraperitoneal injection and PX-478 oral given). Combination group (erastin intraperitoneal injection and PX-478 oral given) had an obviously better anticancer activity than erastin group. However, Lv-GPX4 group (injection with GPX4 overexpression cells, erastin intraperitoneal injection and PX-478 oral given) showed no anticancer effect measured by tumor volume and tumor weight in subcutaneous xenograft model (Fig. 6A-C). Similar results were obtained in orthotopic xenograft model measured by luciferase activity (Fig. 6D-E). The overall survival of combination group was also higher than erastin group (Fig. 6F). Furthermore, the histological analysis indicated that PX-478 group and combination group exhibited weaker immunoreactivity for GPX4 and Lv-GPX4 could efficiently increase GPX4 expression (Fig. 6G). Collectively, PX-478 had very little therapeutic effect on glioblastoma, but it can greatly enhance the anticancer effect of erastin in both subcutaneous xenograft and orthotopic xenograft mouse model.

\section{Discussion}

The incidence of glioblastoma continues to increase globally with the aging of the world's population and glioblastoma remains to have a very low 5-year overall survival rate of only $6.8 \%$ [32]. Tumor cells can develop resistant to various drugs during therapy process including drugs based on ferroptosis [33]. Therapies underlying ferroptosis attracted much attention with high expectations in recent years [34]. Our work has first documented that hypoxia for at least 16 hours can enhance glioblastoma resistance to erastin-induced ferroptosis. Therefore, a better understanding of the mechanism underlying erastinresistance may benefit ferroptosis-targeted treatment. We found one of the most important underlying mechanism of erastin resistance was the up regulation of GPX4, which functioned as the primary enzyme that prevented ferroptosis [35]. Other genes such as STEAP3 and CA9 uncovered by PCR array may also participated in the erastin resistance induced by hypoxia, but GPX4 expressed stable in different types of cells and was considered as the most crucial gene.

PI3K/AKT signaling pathway plays a central role in cell survival [30]. The allosteric AKT inhibitor MK2206 has been reported to decrease tumor growth and enhance the antitumor efficacy of chemotherapeutic agents [36-37]. We found that activated PI3K/AKT/HIF-1 $\mathrm{a}$ axis by hypoxia was essential for the upregulation of GPX4. Both AKT inhibitor MK-2206 and HIF-1a inhibitor PX-478 could significantly inhibit GPX4 expression under hypoxic environment. These results suggested the up-regulation of GPX4 induced by hypoxia was dependent on the activation of PI3K/AKT/HIF-1 $a$ axis.

HIF-1a was a famous transcription factor and regulated multiple environmental adaption genes, but there was no passage about the relationship between HIF-1a and any ferroptosis-related genes. Here, we first found HIF-1a directly regulated GPX4 transcription by binding to its promoter region $-131 \mathrm{bp}$ to $1 \mathrm{bp}$. HIF1a inhibitor PX-478 had antitumor effect with fewer side effect, but the effect of PX-478 alone was limited 
[38]. Scientists paid much attention on the synergetic effect of PX-478 including combination with radiotherapy or immunotherapy [39-40]. Radiotherapy and immunotherapy were related to ferroptosis [41-42]. Given that HIF-1a regulated ferroptosis core gene GPX4, we hypothesized HIF-1a inhibitor PX478 played a vital role in promoting ferroptosis by inhibiting GPX4 expression. We found cells treated with PX-478 exhibited higher lipid peroxidation level by C11-BODIPY staining. We also found deeply stained, shrunken mitochondria by transmission electron microscopy.

Tumor microenvironment was featured with regional hypoxia [43]. So, we hypothesized the regional hypoxia of tumor microenvironment in vivo strengthened the erastin-resistance by HIF-1a/GPX4 axis. We employed subcutaneous xenograft and orthotopic xenograft mouse model to test the function of PX-478 in erastin therapy. We obtained ideal experimental results, which showed PX-478 can effectively decreased the GPX4 expression level in tumor tissue and promoted the anticancer effect of erastin.

To our best knowledge, this is the first study to explore the role of hypoxia in erastin resistance in glioblastoma. And this is the first study to uncover the relationship between PI3K/AKT/HIF-1a pathway and ferroptosis core gene GPX4. We first put forward that combination of erastin and PX-478 had a good effect on anticancer effect. However, we did not test other ferroptosis-related therapy. Besides, we had not focused on patients, because erastin had limitations when used in human because of its low solubility in water and side effects.

\section{Conclusion}

In conclusion, we demonstrated that hypoxia enhanced glioblastoma resistance to erastin-induced ferroptosis by activating the PI3K/AKT/HIF-1a pathway and promoting GPX4 expression (FIG. 7). We proved PX-478 and erastin had a coordinated intensification effect on anticancer activity in vitro and in vivo. Future work should include other kinds of ferroptosis-related therapies to evaluate whether PX-478 not only promotes erastin but also others especially those sufficiently safe for the treatment of human tumor.

\section{Abbreviations}

TME: the tumor microenvironment; GPX4: glutathione peroxidase 4; HIF: hypoxia-inducible factor; DMEM: Dulbecco's modified Eagle's medium; FBS: fetal bovine serum; CCK-8: Cell Counting Kit-8; IC50: Half-maximal inhibitory concentration assay; ECL: electrochemiluminescence; IF:

Immunofluorescence; IHC: Immunohistochemistry; MDA: Malondialdehyde; STEAP3: STEAP3 metalloreductase; CA9: carbonic anhydrase 9.

\section{Declarations}

\section{Ethics approval and consent to participate}


The protocol of this study was approved by the Ethics and Human Subject Committee of Shandong Provincial Hospital, and all experiments and methods met the standards of the relevant guidelines and regulations.

\section{Consent for publication}

Not applicable.

\section{Availability of data and materials}

The datasets used and analyzed during the current study are available from the corresponding author on reasonable request.

\section{Competing interests}

The authors declare that they have no competing interests

\section{Funding}

This work was supported by the National Natural Science Foundation of China (Grant number 81771270).

\section{Authors' contributions}

SC and CF performed the original draft preparation, revision, created the tables and figures, and were the major contributors in writing the manuscript. $\mathrm{BP}, \mathrm{TH}, \mathrm{XS}$ and $\mathrm{DH}$ made suggestions to the writing of the manuscript and revisions to tables and figures. RZ and QP participated in conceptualization and methodology. All authors read and approved the final manuscript.

\section{Acknowledgements}

Not applicable.

\section{References}

1. Touat M, Idbaih A, Sanson M, Ligon KL. Glioblastoma targeted therapy: updated approaches from recent biological insights. Ann Oncol. 2017; 28: 1457-1472.

2. Mudassar F, Shen H, O'Neill G, Hau E. Targeting tumor hypoxia and mitochondrial metabolism with anti-parasitic drugs to improve radiation response in high-grade gliomas. J Exp Clin Cancer Res. 2020; 39: 208.

3. Tan AC, Ashley DM, López GY, Malinzak M, Friedman HS, Khasraw M. Management of glioblastoma: State of the art and future directions. CA Cancer J Clin. 2020; 70: 299-312.

4. Alifieris C, Trafalis DT. Glioblastoma multiforme: Pathogenesis and treatment. Pharmacol Ther. 2015; 152: 63-82. 
5. Jing X, Yang F, Shao C, Wei K, Xie M, Shen H, et al. Role of hypoxia in cancer therapy by regulating the tumor microenvironment. Molecular cancer. 2019; 18: 157.

6. Mertens C, Mora J, Ören B, Grein S, Winslow S, Scholich K, et al. Macrophage-derived lipocalin-2 transports iron in the tumor microenvironment. Oncoimmunology. 2018; 7: e1408751.

7. Wang P, Yan Q, Liao B, Zhao L, Xiong S, Wang J, et al. The HIF1a/HIF2a-miR210-3p network regulates glioblastoma cell proliferation, dedifferentiation and chemoresistance through EGF under hypoxic conditions. Cell Death Dis. 2020; 11: 992.

8. Cowman S, Fan YN, Pizer B, Sée V. Decrease of Nibrin expression in chronic hypoxia is associated with hypoxia-induced chemoresistance in some brain tumour cells. BMC Cancer. 2019; 19: 300.

9. Marampon F, Gravina GL, Zani BM, Popov VM, Fratticci A, Cerasani M, et al. Hypoxia sustains glioblastoma radioresistance through ERKs/DNA-PKcs/HIF-1 a functional interplay. Int J Oncol. 2014; 44: 2121-2131.

10. Catalina-Rodriguez O, Kolukula VK, Tomita Y, Preet A, Palmieri F, Wellstein A, et al. The mitochondrial citrate transporter, $\mathrm{CIC}$, is essential for mitochondrial homeostasis. Oncotarget. 2012; 3: 1220-1235.

11. Graham K, Unger E. Overcoming tumor hypoxia as a barrier to radiotherapy, chemotherapy and immunotherapy in cancer treatment. Int J Nanomedicine. 2018; 13: 6049-6058.

12. Schulz A, Meyer F, Dubrovska A, Borgmann K. Cancer Stem Cells and Radioresistance: DNA Repair and Beyond. Cancers (Basel). 2019; 11.

13. Liu J, Gao L, Zhan N, Xu P, Yang J, Yuan F, et al. Hypoxia induced ferritin light chain (FTL) promoted epithelia mesenchymal transition and chemoresistance of glioma. J Exp Clin Cancer Res. 2020; 39: 137.

14. Persano L, Rampazzo E, Basso G, Viola G. Glioblastoma cancer stem cells: role of the microenvironment and therapeutic targeting. Biochem Pharmacol. 2013; 85: 612-622.

15. Uribe D, Torres Á, Rocha JD, Niechi I, Oyarzún C, Sobrevia L, et al. Multidrug resistance in glioblastoma stem-like cells: Role of the hypoxic microenvironment and adenosine signaling. $\mathrm{Mol}$ Aspects Med. 2017; 55: 140-151.

16. Majmundar AJ, Wong WJ, Simon MC. Hypoxia-inducible factors and the response to hypoxic stress. Mol Cell. 2010; 40: 294-309.

17. Lee JW, Ko J, Ju C, Eltzschig HK. Hypoxia signaling in human diseases and therapeutic targets. Experimental \& molecular medicine. 2019; 51: 1-13.

18. Koh MY, Spivak-Kroizman T, Venturini S, Welsh S, Williams RR, Kirkpatrick DL, et al. Molecular mechanisms for the activity of PX-478, an antitumor inhibitor of the hypoxia-inducible factor-1alpha. Mol Cancer Ther. 2008; 7: 90-100.

19. Palayoor ST, Mitchell JB, Cerna D, Degraff W, John-Aryankalayil M, Coleman CN. PX-478, an inhibitor of hypoxia-inducible factor-1 alpha, enhances radiosensitivity of prostate carcinoma cells. International journal of cancer. 2008; 123: 2430-2437. 
20. Lang M, Wang X, Wang H, Dong J, Lan C, Hao J, et al. Arsenic trioxide plus PX-478 achieves effective treatment in pancreatic ductal adenocarcinoma. Cancer letters. 2016; 378: 87-96.

21. Huang R, Dong R, Wang N, He Y, Zhu P, Wang C, et al. Adaptive Changes Allow Targeting of Ferroptosis for Glioma Treatment. Cell Mol Neurobiol. 2021.

22. Conrad M, Pratt DA. The chemical basis of ferroptosis. Nature chemical biology. 2019; 15: 11371147.

23. Mao C, Liu X, Zhang Y, Lei G, Yan Y, Lee H, et al. DHODH-mediated ferroptosis defence is a targetable vulnerability in cancer. Nature. 2021; 593: 586-590.

24. Zhao Y, Li Y, Zhang R, Wang F, Wang T, Jiao Y. The Role of Erastin in Ferroptosis and Its Prospects in Cancer Therapy. Onco Targets Ther. 2020; 13: 5429-5441.

25. Chen L, Li X, Liu L, Yu B, Xue Y, Liu Y. Erastin sensitizes glioblastoma cells to temozolomide by restraining XCT and cystathionine-y-lyase function. Oncology reports. 2015; 33: 1465-1474.

26. Brennan CW, Verhaak RG, McKenna A, Campos B, Noushmehr H, Salama SR, et al. The somatic genomic landscape of glioblastoma. Cell. 2013; 155: 462-477.

27. Yang Y, Luo M, Zhang K, Zhang J, Gao T, Connell DO, et al. Nedd4 ubiquitylates VDAC2/3 to suppress erastin-induced ferroptosis in melanoma. Nat Commun. 2020; 11: 433.

28. Gai C, Liu C, Wu X, Yu M, Zheng J, Zhang W, et al. MT1DP loaded by folate-modified liposomes sensitizes erastin-induced ferroptosis via regulating miR-365a-3p/NRF2 axis in non-small cell lung cancer cells. Cell Death Dis. 2020; 11: 751.

29. Torrisi F, Vicario N, Spitale FM, Cammarata FP, Minafra L, Salvatorelli L, et al. The Role of Hypoxia and SRC Tyrosine Kinase in Glioblastoma Invasiveness and Radioresistance. Cancers (Basel). 2020; 12.

30. Huang W, Ding X, Ye H, Wang J, Shao J, Huang T. Hypoxia enhances the migration and invasion of human glioblastoma U87 cells through PI3K/Akt/mTOR/HIF-1a pathway. Neuroreport. 2018; 29: 1578-1585.

31. Yi J, Zhu J, Wu J, Thompson CB, Jiang X. Oncogenic activation of PI3K-AKT-mTOR signaling suppresses ferroptosis via SREBP-mediated lipogenesis. Proceedings of the National Academy of Sciences of the United States of America. 2020; 117: 31189-31197.

32. Wen PY, Weller M, Lee EQ, Alexander BM, Barnholtz-Sloan JS, Barthel FP, et al. Glioblastoma in adults: a Society for Neuro-Oncology (SNO) and European Society of Neuro-Oncology (EANO) consensus review on current management and future directions. Neuro-oncology. 2020; 22: 10731113.

33. Sazonova EV, Kopeina GS, Imyanitov EN, Zhivotovsky B. Platinum drugs and taxanes: can we overcome resistance? Cell death discovery. 2021; 7: 155.

34. Su Y, Zhao B, Zhou L, Zhang Z, Shen Y, Lv H, et al. Ferroptosis, a novel pharmacological mechanism of anti-cancer drugs. Cancer letters. 2020; 483: 127-136. 
35. Yang WS, SriRamaratnam R, Welsch ME, Shimada K, Skouta R, Viswanathan VS, et al. Regulation of ferroptotic cancer cell death by GPX4. Cell. 2014; 156: 317-331.

36. Liu J, Duan Z, Guo W, Zeng L, Wu Y, Chen Y, et al. Targeting the BRD4/FOXO3a/CDK6 axis sensitizes AKT inhibition in luminal breast cancer. Nat Commun. 2018; 9: 5200.

37. Hirai H, Sootome H, Nakatsuru Y, Miyama K, Taguchi S, Tsujioka K, et al. MK-2206, an allosteric Akt inhibitor, enhances antitumor efficacy by standard chemotherapeutic agents or molecular targeted drugs in vitro and in vivo. Mol Cancer Ther. 2010; 9: 1956-1967.

38. Lee K, Kim HM. A novel approach to cancer therapy using PX-478 as a HIF-1a inhibitor. Archives of pharmacal research. 2011; 34: 1583-1585.

39. Ban HS, Uto Y, Nakamura H. Hypoxia-inducible factor inhibitors: a survey of recent patented compounds (2004 - 2010). Expert opinion on therapeutic patents. 2011; 21: 131-146.

40. Jiang M, Qin B, Luo L, Li X, Shi Y, Zhang J, et al. A clinically acceptable strategy for sensitizing antiPD-1 treatment by hypoxia relief. Journal of controlled release : official journal of the Controlled Release Society. 2021; 335: 408-419.

41. Zhu X, Zhou Y, Ou Y, Cheng Z, Han D, Chu Z, et al. Characterization of ferroptosis signature to evaluate the predict prognosis and immunotherapy in glioblastoma. Aging. 2021; 13: 17655-17672.

42. Lei $G$, Zhang $Y$, Hong $T$, Zhang $X$, Liu $X$, Mao $C$, et al. Ferroptosis as a mechanism to mediate p53 function in tumor radiosensitivity. Oncogene. 2021; 40: 3533-3547.

43. Heddleston JM, Hitomi M, Venere M, Flavahan WA, Yang K, Kim Y, et al. Glioma stem cell maintenance: the role of the microenvironment. Current pharmaceutical design. 2011; 17: 2386-2401.

\section{Figures}


(A)

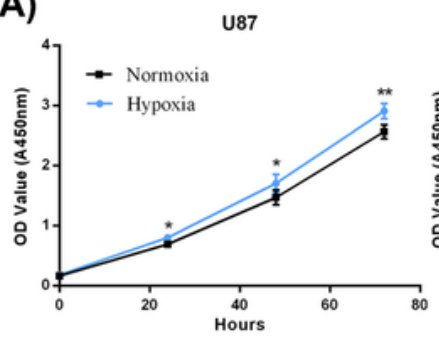

(C)

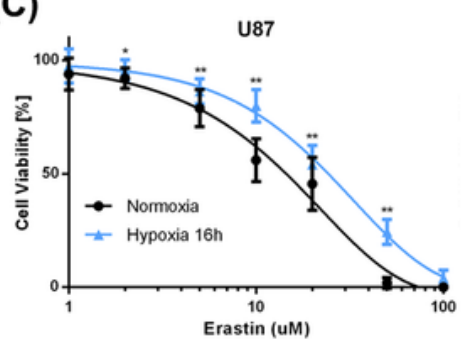

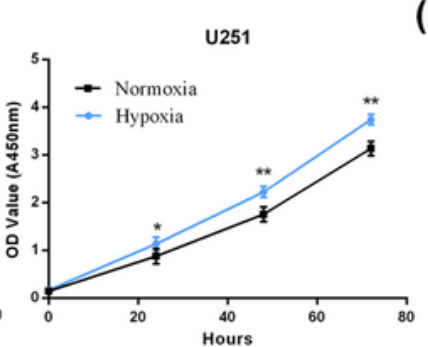

U251

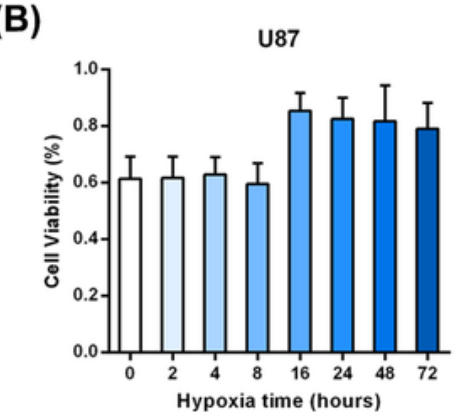

(D)
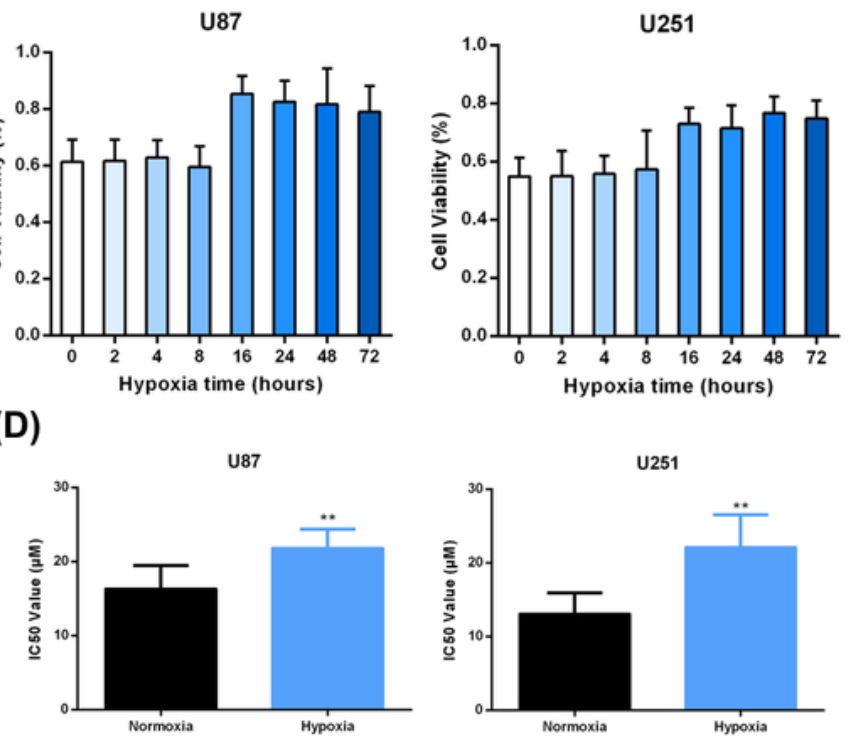

(E)
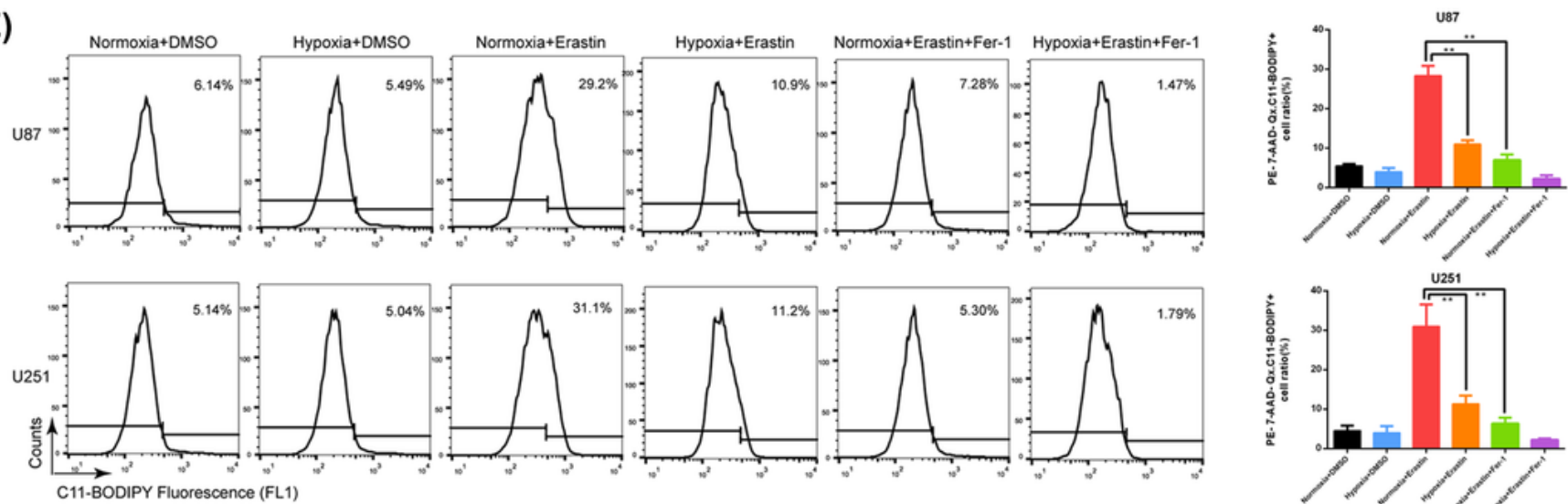

(F)
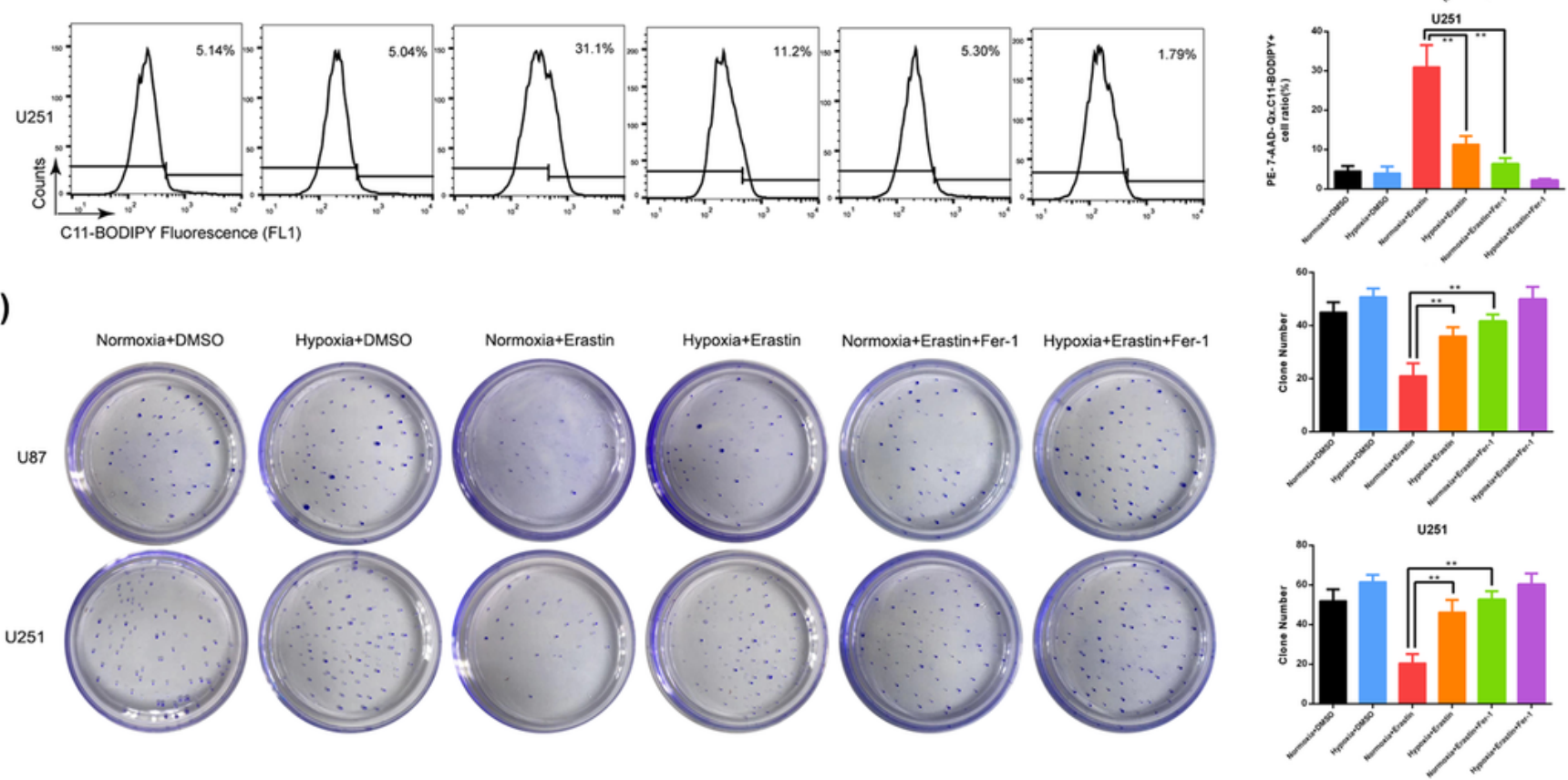

\section{Figure 1}

Hypoxia enhanced resistance of glioblastoma cells to erastin-induced ferroptosis $A$. The growth curves of U87 and U251 cells were examined using a CCK-8 assay. Cell growth was promoted under hypoxic condition. B. Cell resistance changes to erastin at a series of time points after hypoxic incubation in U87 and U251 cells. Glioblastoma cells under hypoxic environment for at least 16 hours showed stronger resistance. C. Cell viability curves at different concentrations of erastin for $24 \mathrm{~h}$. Glioblastoma cells under 
hypoxic environment became less sensitive to erastin. D. Half-maximal inhibitory concentrations (IC50) were calculated in each group. Hypoxic cells had an obvious higher IC50 to erastin. E. Lipid peroxidation assessed in U87 and U251 cells after exposure to hypoxia, erastin, and fer-1 by flow cytometry using C11BODIPY. F. Clone formation assay of cancer cells in absence or presence of $10 \mu \mathrm{M}$ erastin or $0.5 \mu \mathrm{M}$ fer- 1 under normoxia or hypoxia. The bar graph showed mean \pm SD of 3 independent experiments. ${ }^{*} P<0.05$, $* * \mathrm{P}<0.01$.

(A)

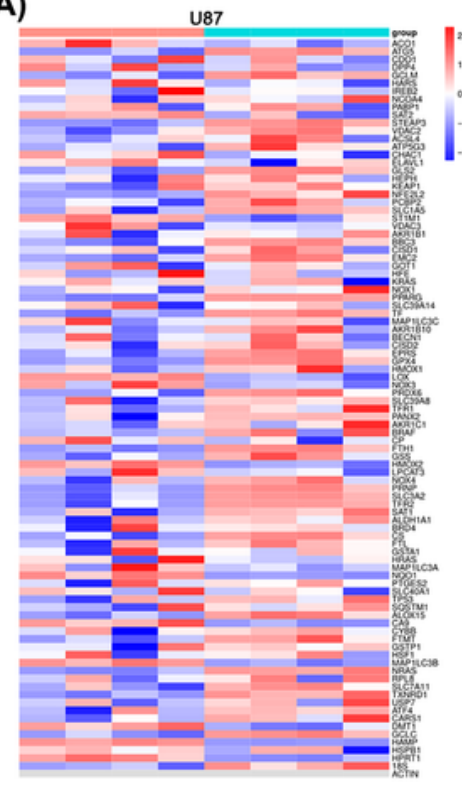

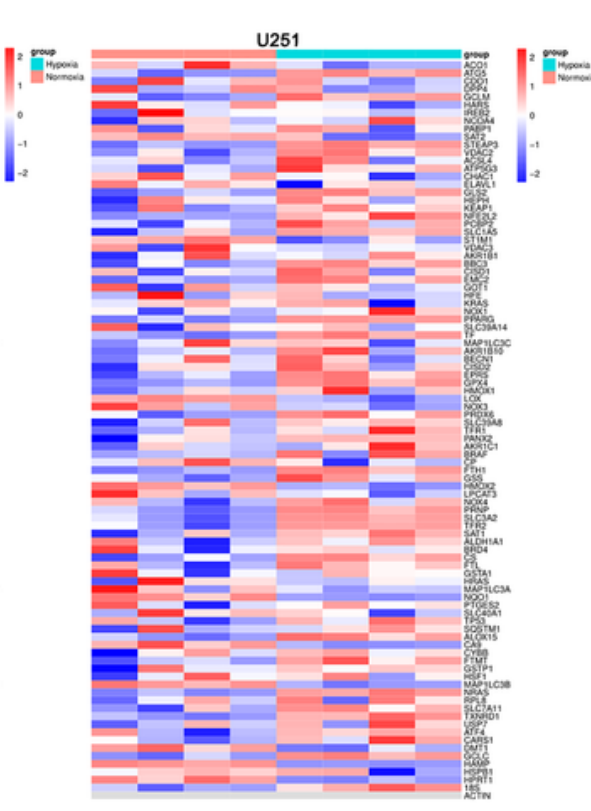

(B)
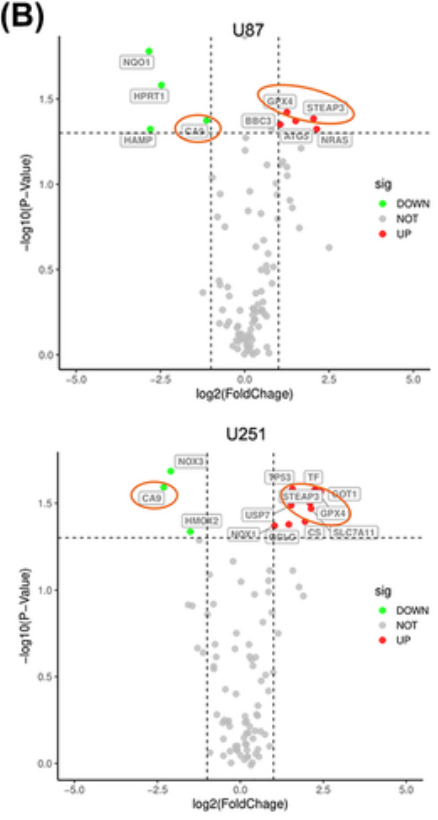

(C)
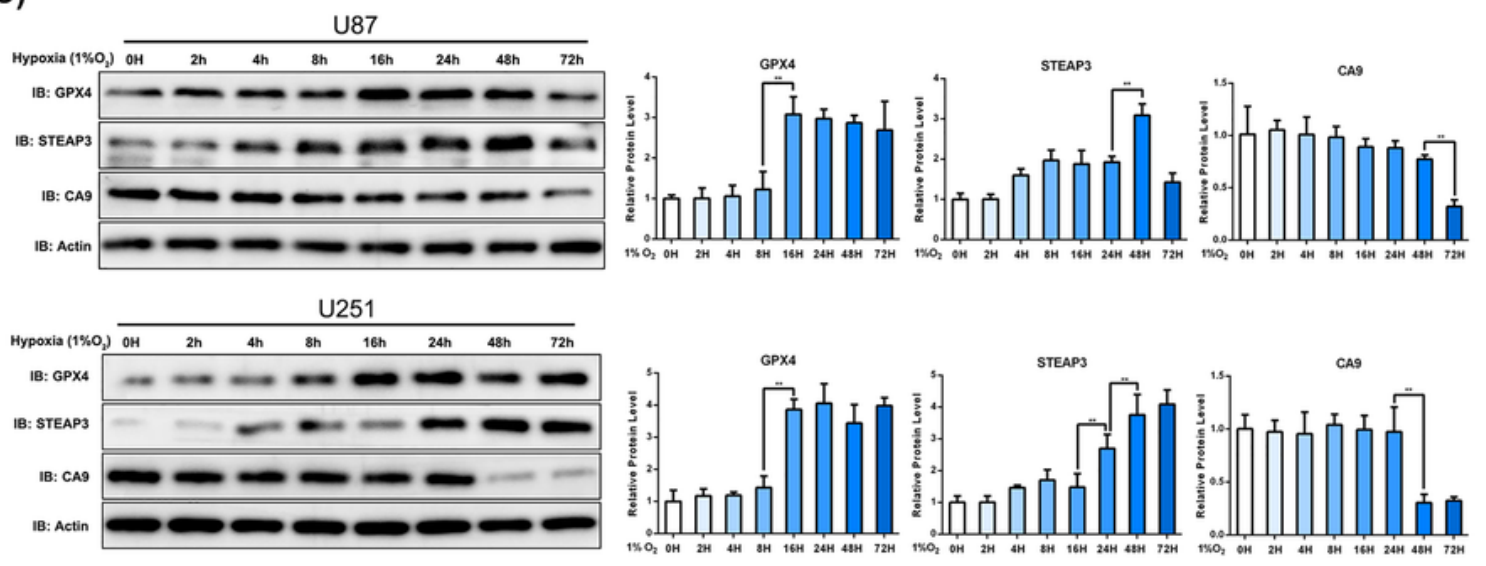

(D)
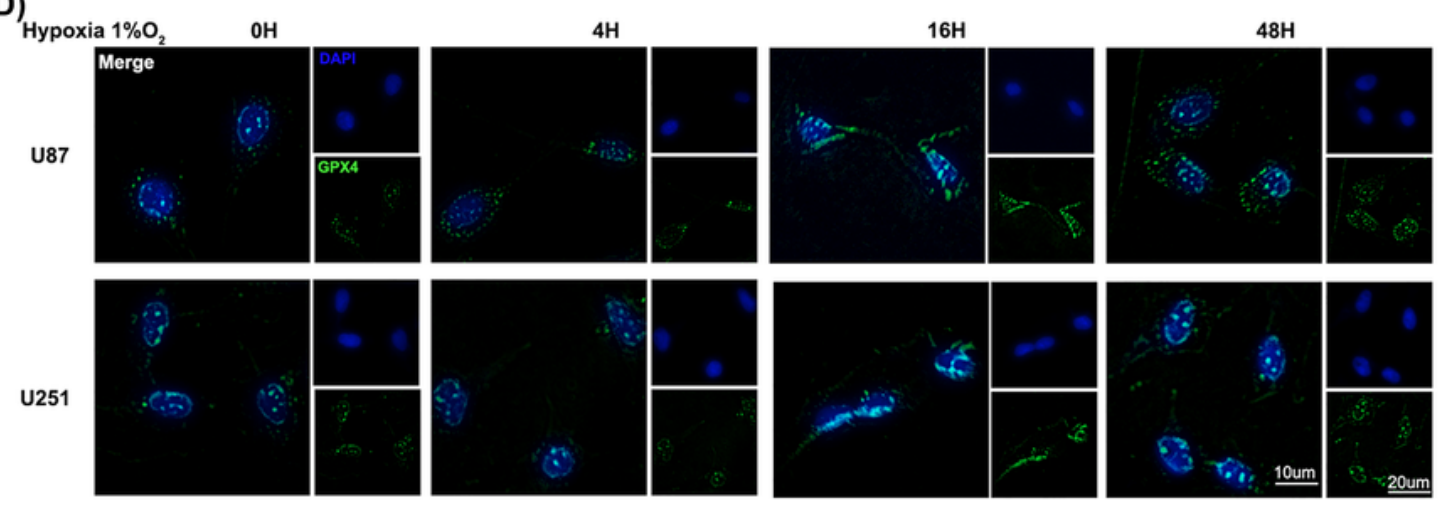

Figure 2 
GPX4 was screened out as a potential effector molecular of hypoxia A. Ferroptosis-related qPCR array of normoxia and hypoxia identified several differentially expressed genes in U87 and U251 cells. B. The volcano map of differentially expressed genes depicted by difference degree and p-value. GPX4, STEAP3 and CA9 were picked out. C. Protein expression level of GPX4, STEAP3 and CA9 over a series of time points measured by western blot. GPX4 protein expression increased significantly at the 16th hour under hypoxia. D. GPX4 protein location and expression level uncovered by Immunofluorescence. Higher GPX4 protein level after at least 16 hours hypoxic incubation but no apparent location changes. The bar graph showed mean \pm SD of 3 independent experiments. ${ }^{*} P<0.05, * * P<0.01$.

(A)

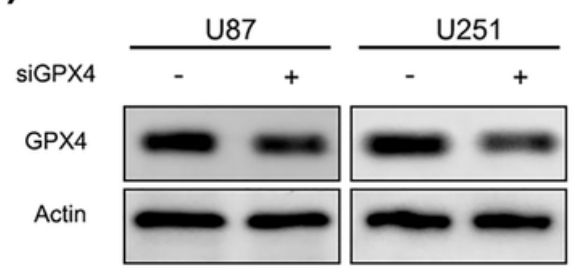

(B)

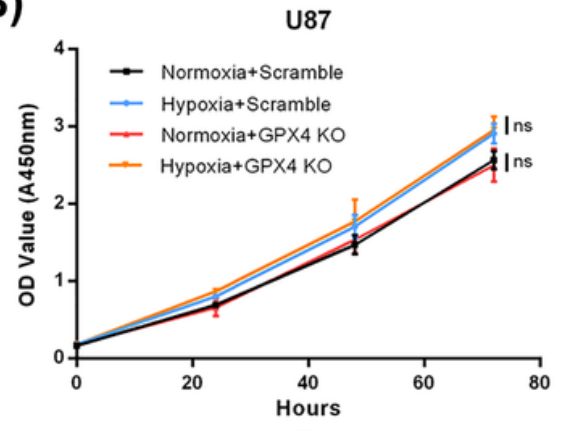

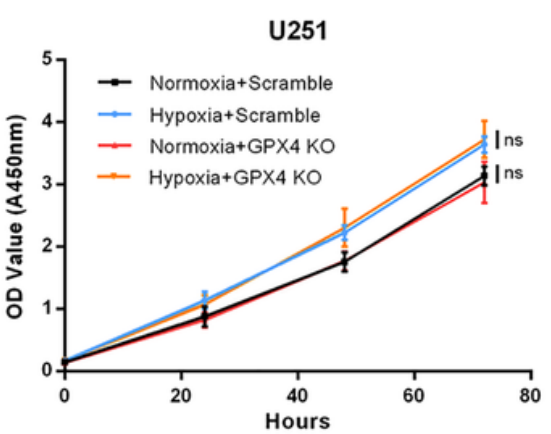

(D)

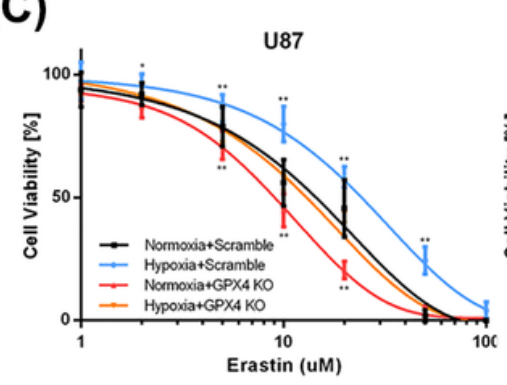

(E)

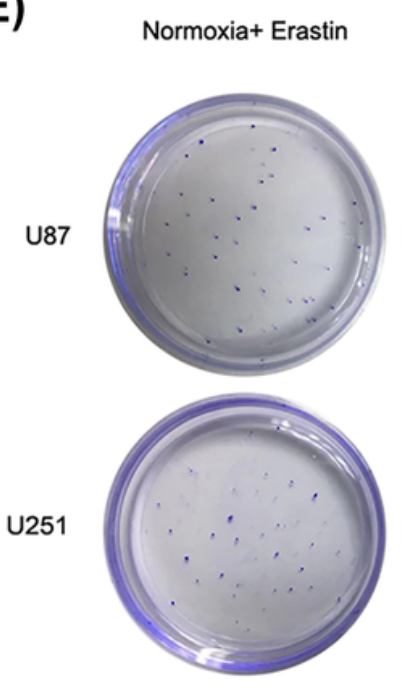

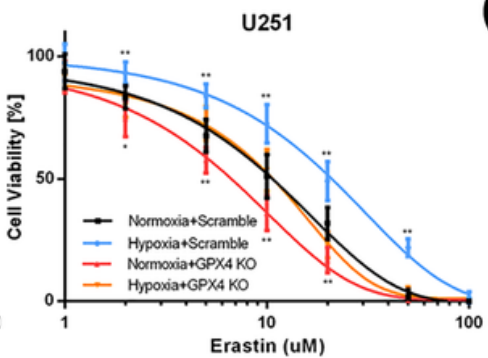

Erastin (uM)

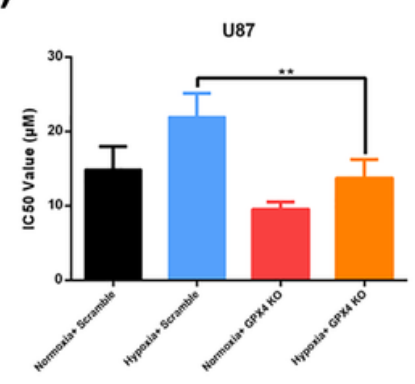

Hypoxia+ GPX4 KO + Erastin

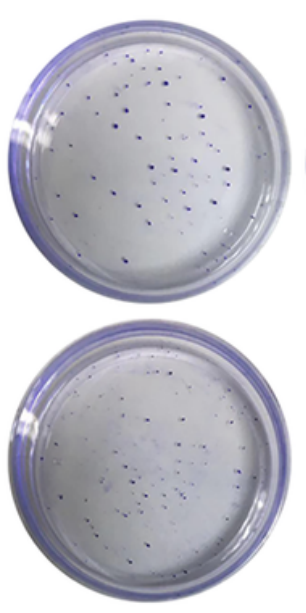

Normoxia+ GPX4 KO + Erastin

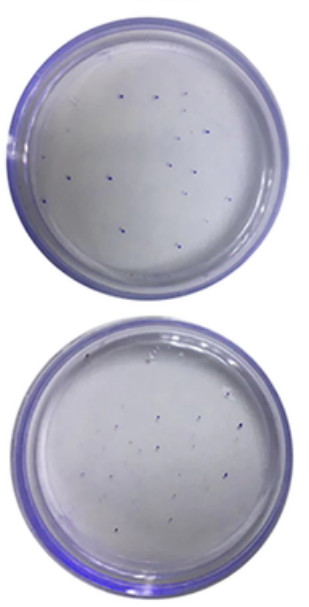

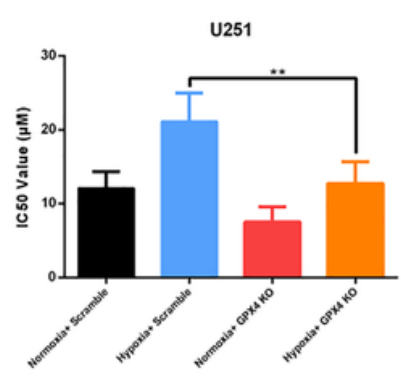

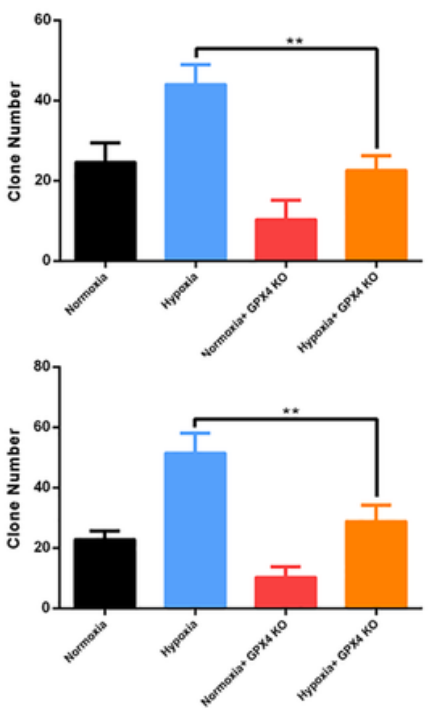

Figure 3 
Hypoxia promoted erastin resistance via increasing GPX4 expression A. GPX4 protein expression level. GPX4 KO can significantly inhibit the protein expression level. B. The growth curves were examined using a CCK-8 assay. GPX4 knockout did not influence the cell proliferation rate of glioblastoma cells. C. Cell viability curves at different concentrations of erastin for $24 \mathrm{~h}$. GPX 4 knockout could reverse the enhanced erastin-resistance induced by hypoxia. D. The IC50 of erastin in GPX4 knockout group was obviously low than that of scramble group under hypoxic or normoxic condition. E. Clone formation assay of cancer cells in absence or presence of $10 \mu \mathrm{M}$ erastin for 2 weeks. GPX4 knockout could reverse the enhanced cell colony formation ability induced by hypoxia. The bar graph showed mean \pm SD of 3 independent experiments. ${ }^{\star} \mathrm{P}<0.05,{ }^{\star *} \mathrm{P}<0.01$. 
(A)

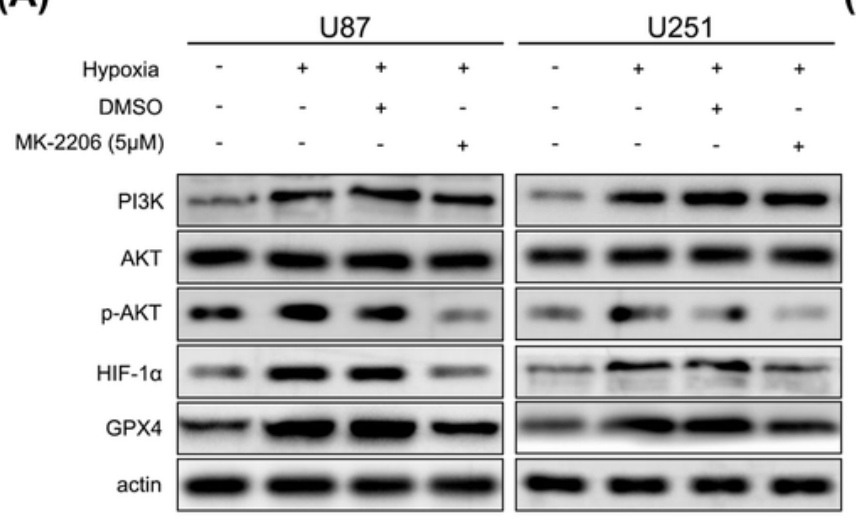

(C)

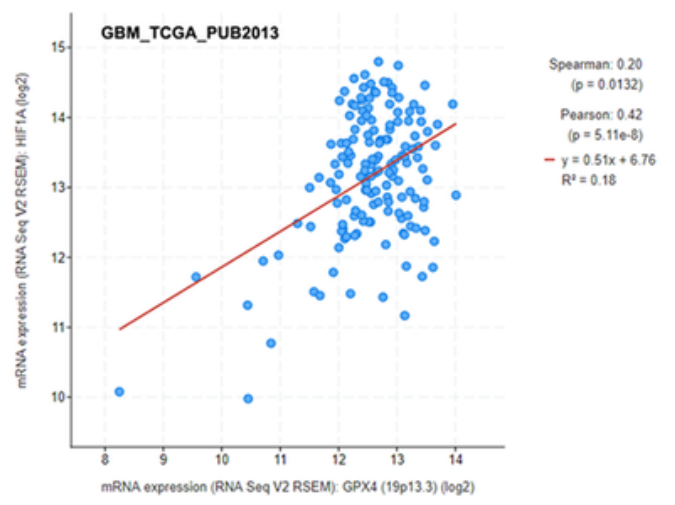

(E)

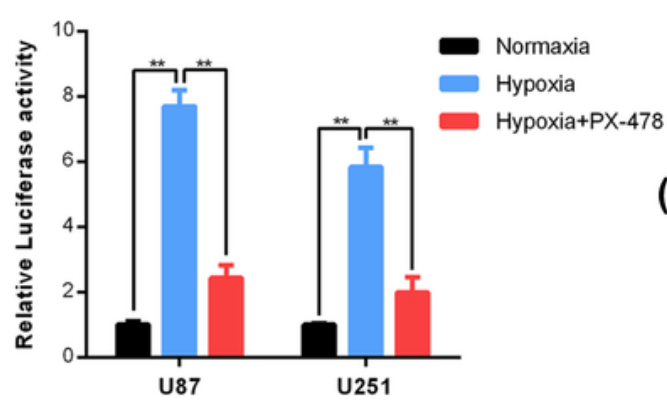

(G)
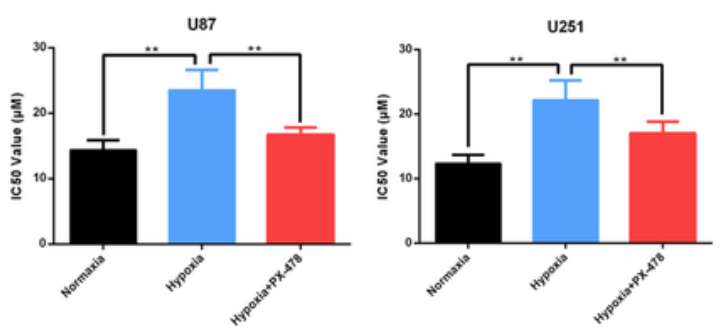

(B)
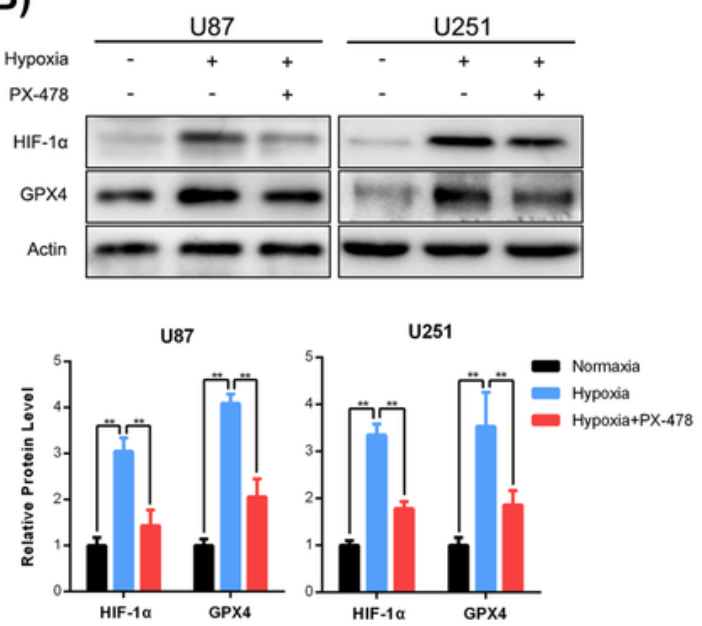

(D)

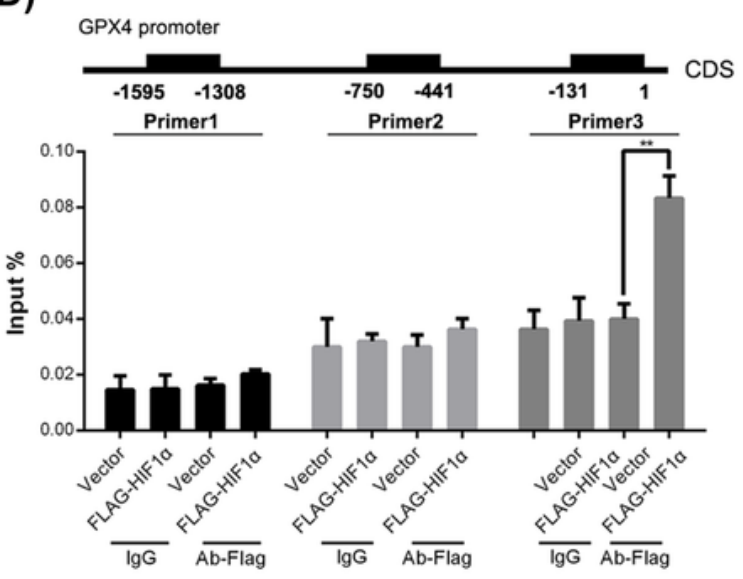

(F)
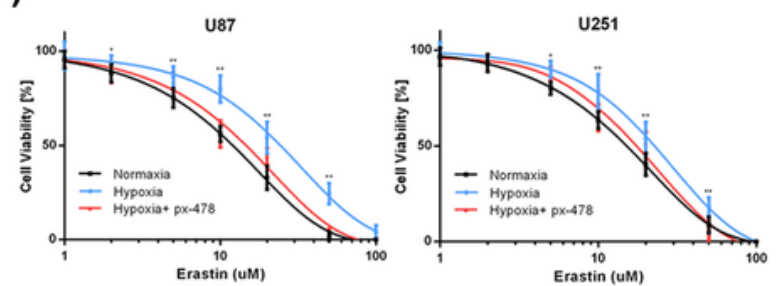

\section{Figure 4}

Hypoxia promoted GPX4 expression via PI3K/AKT/HIF-1 a pathway A. Protein expression level of PI3K, AKT, p-AKT, HIF-1 a and GPX4 influenced by oxygen content and AKT inhibitor MK-2206 measured by western blot. AKT inhibitor MK-2206 along with hypoxia attenuated the expression level of p- AKT, HIF-1a and GPX4. B. Protein expression level of HIF-1a and GPX4 influenced by oxygen content and HIF-1a inhibitor PX-478 measured by western blot. PX-478 markedly decreased HIF-1a and GPX4 protein levels. 
C. The relationship between HIF-1a and GPX4 expression level in TCGA. GPX4 was positively correlated with HIF-1a in 257 glioblastoma tumor samples. D. Bar plot representing qPCR values of the ChIP results. HIF-1a can indeed bind to the GPX4 promoter region $-131 \mathrm{bp}$ to $1 \mathrm{bp}$. E. Bar plots of relative luciferase activity under normoxia condition, hypoxia condition, and hypoxia with PX-478 condition. Hypoxia increased luciferase activity and hypoxia with PX-478 reversed it. F. Cell viability curves at different concentrations of erastin for $24 \mathrm{~h}$. PX-478 can reverse the enhanced erastin-resistance induced by hypoxia. G. The IC50 of erastin in hypoxia with PX-478 group was obviously low than that of hypoxia group. The bar graph showed mean \pm SD of 3 independent experiments. ${ }^{*} P<0.05,{ }^{*} P<0.01$. 


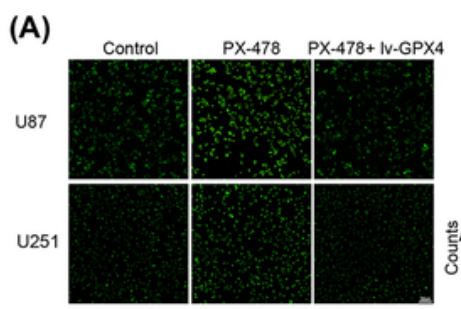

(B)

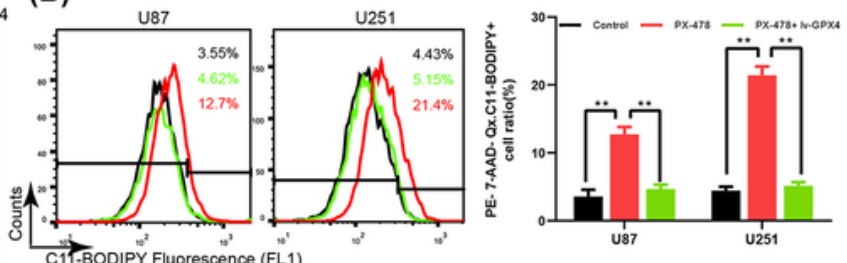

(C)

(D)

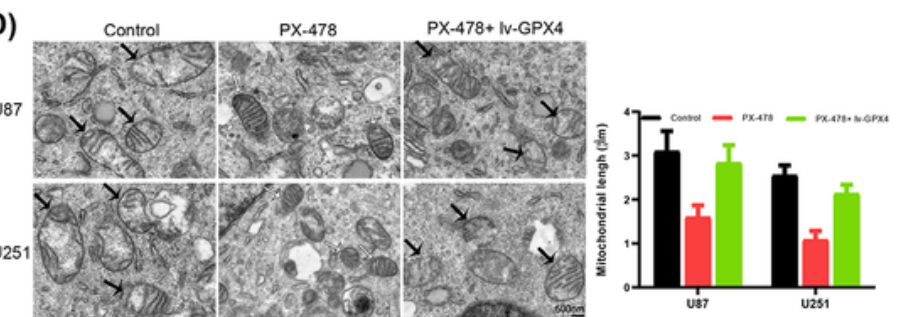

(E)
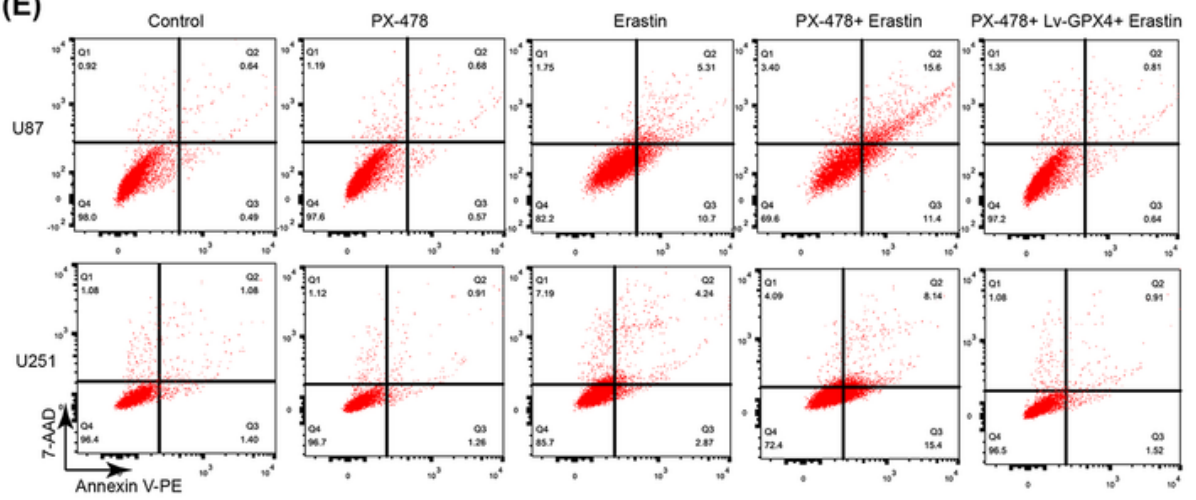

(F)
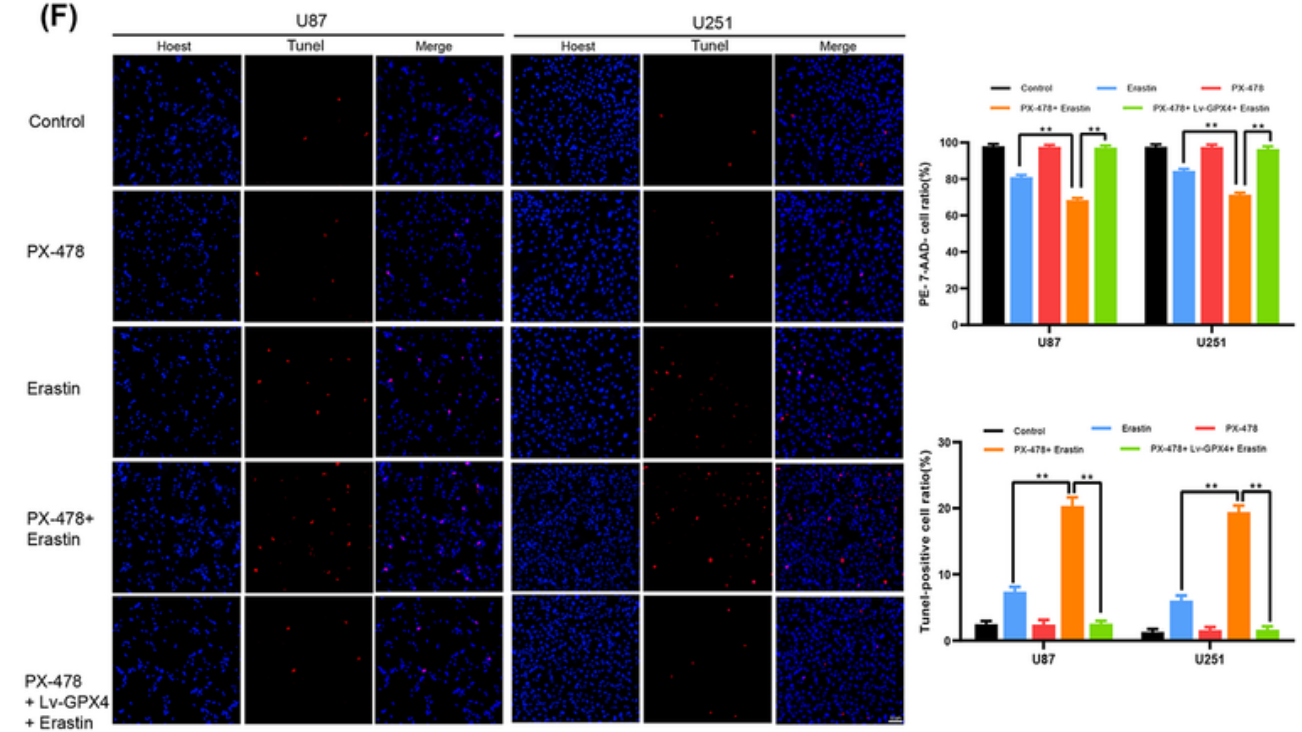

Figure 5

PX-478 induced lipid peroxidation in U87 and U251 cells. A-B. Lipid peroxidation assessment in U87 and

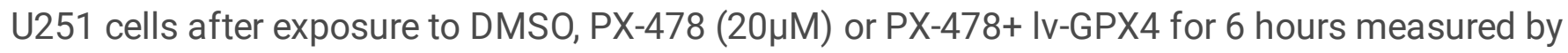
fluorescence microscope (A) and flow cytometry (B). C. Quantitative analysis of the expression levels of MDA in U87 and U251 cells after exposure to DMSO, PX-478 or PX-478+ Iv-GPX4 for 6 hours. MDA increased after treating with PX-478 and reversed in the presence of Lv-GPX4. D. Transmission electron 
microscopy images of U87 and U251 cells after exposure to DMSO, PX-478, or PX-478+ Iv-GPX4. PX-478 treated cancer cells exhibited shrunken mitochondria with enhanced membrane density. Black arrow: normal mitochondria; White arrow: shrunken and high membrane density mitochondria. Quantitative analysis of mitochondrial length was listed on the right. E-F. Cell apoptosis was detected by flow cytometry (E) and TUNEL fluorescence staining (F) in U87 and U251 cells after exposure to DMSO, PX478 , erastin, PX-478+ erastin and PX-478+ Iv-GPX4+ erastin. The bar graph showed mean \pm SD of 3 independent experiments. ${ }^{*} \mathrm{P}<0.05,{ }^{*} \mathrm{P}<0.01$.

(A)

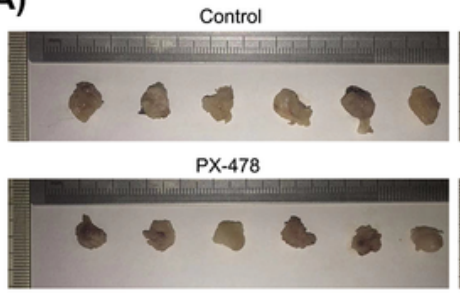

PX-478+ Lv-GPX4+ Erastin

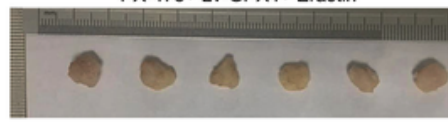

(D)

Control

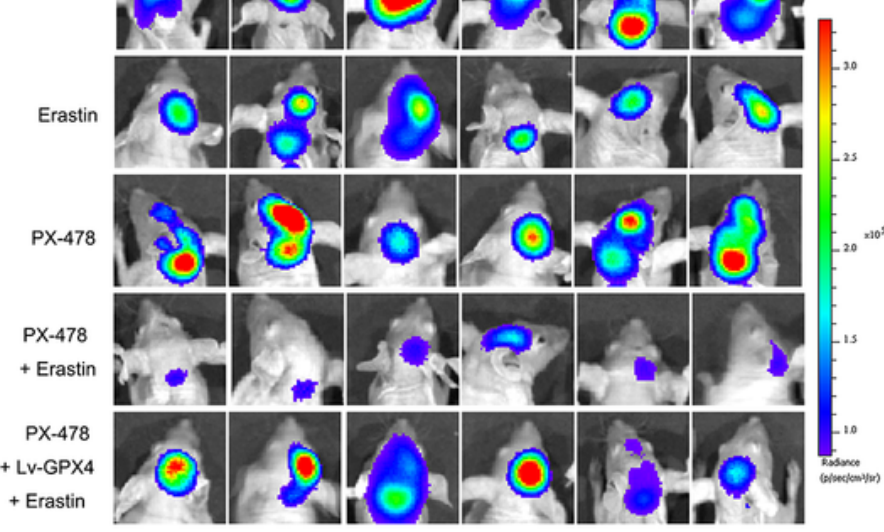

(B)
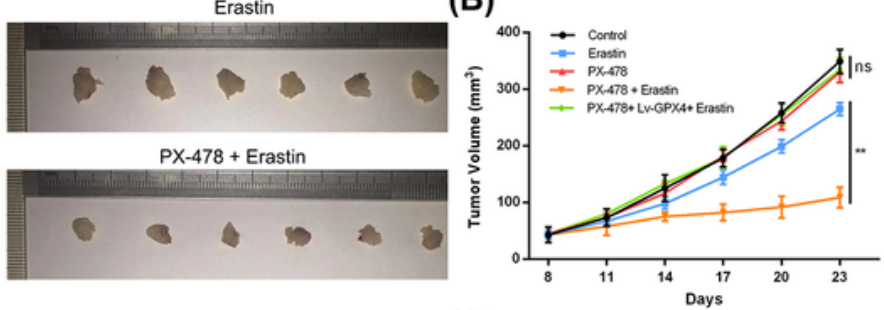

(C)

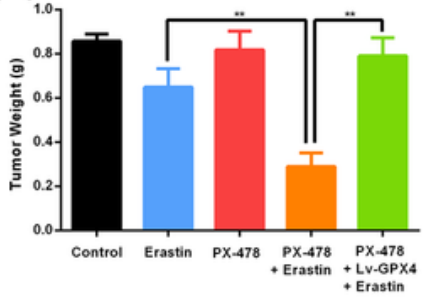

(E)

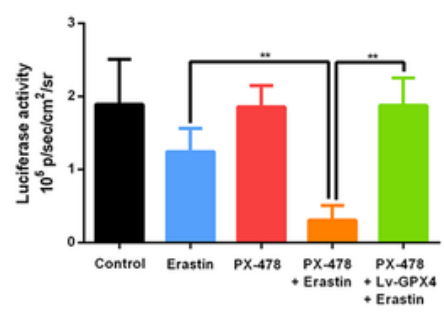

(F)

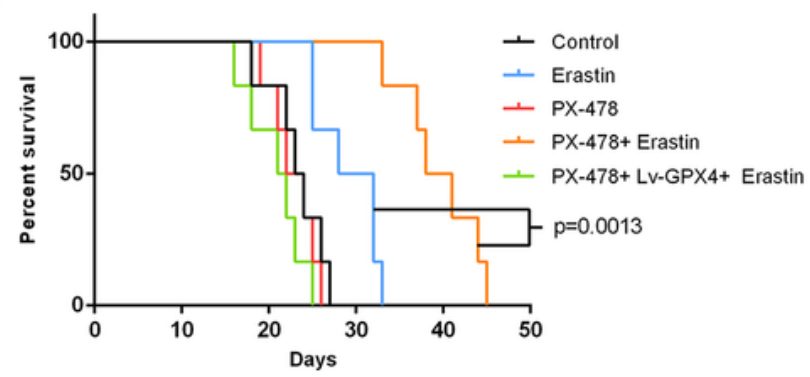

(G)

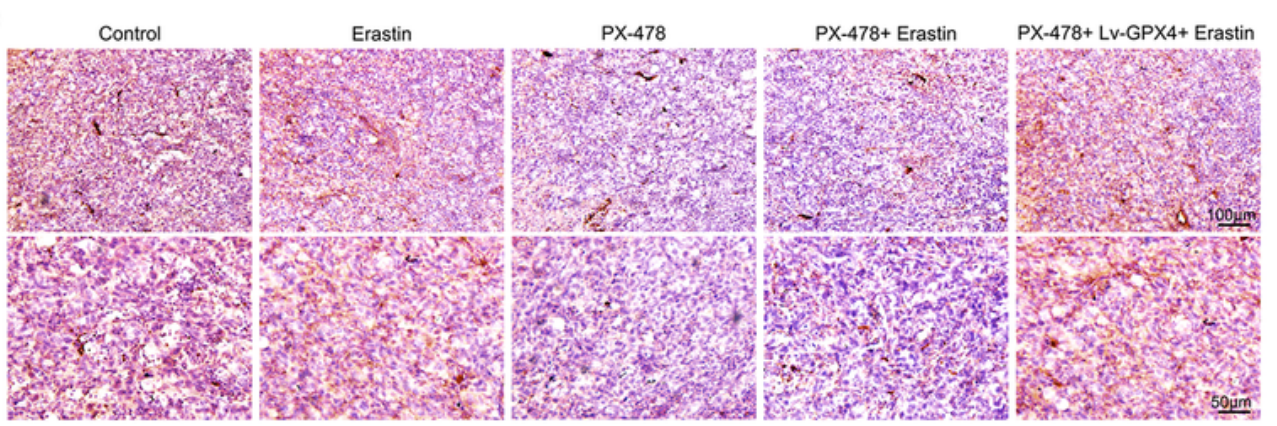


Figure 6

PX-478 promoted the anticancer activity of erastin in vivo A-C. Xenografts derived from U87 or U87/LvGPX4 cells which were treated with different drugs demonstrated tumor volume (B) and weight (C). Erastin+ PX478 had an obviously better anticancer activity than erastin group in U87 cells. Erastin+ PX478 had a weak anticancer activity in U87/Lv-GPX4 cells. D-E. Luciferase activities verified the decreased and increased tumor growth respectively in orthotopic glioblastoma models established by U87 or U87/Lv-GPX4 cells. Similar results were obtained from the five group. F. Curves show the survival rates of the engrafted mice. G. Immunohistochemical labelling showed the downregulated expression of GPX4 after treating with PX-478 and Lv-GPX4 could efficiently increase GPX4 expression in vivo. The bar graph showed mean \pm SD of 3 independent experiments. ${ }^{*} P<0.05,{ }^{*} \mathrm{P}<0.01$.

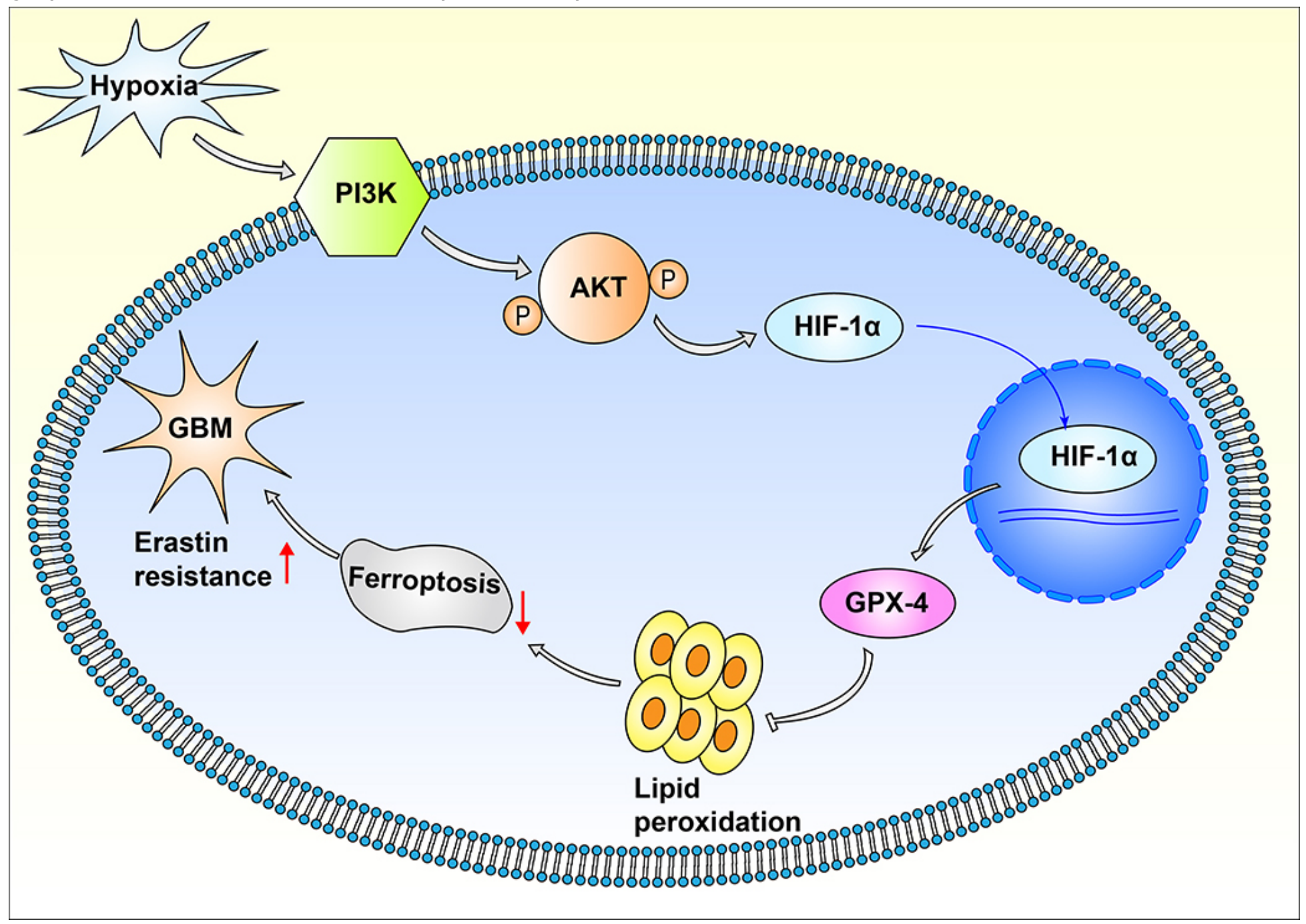

Figure 7

Pattern diagram of hypoxia modulation of ferroptosis. Hypoxia activated PI3K/AKT/HIF-1a pathway in U87 and U251 cells. HIF-1a translocated into nucleus and bound to the GPX4 gene promoter region and promoted GPX4 transcription. Mature GPX4 protein neutralized lipid peroxidation to defend erastininduced ferroptosis. 


\section{Supplementary Files}

This is a list of supplementary files associated with this preprint. Click to download.

- Supplementaryinformation.docx 